SUPPORTING INFORMATION

\title{
EnzyDock: Protein-Ligand Docking of Multiple Reactive States Along a Reaction Coordinate in Enzymes
}

\author{
Susanta Das, ${ }^{\text {a }}$ Mor Shimshi, ${ }^{\text {a }}$ Keren Raz, ${ }^{\mathrm{a}}$ Neta Nitoker, ${ }^{\mathrm{a}}$ Anil Mhashal, ${ }^{\mathrm{a}}$ Tamar Ansbacher, ${ }^{\mathrm{a}, \mathrm{b}}$ Dan T. \\ Major ${ }^{\mathrm{a},}$ \\ ${ }^{a}$ Department of Chemistry, Bar-Ilan University, Ramat-Gan 52900, Israel \\ ${ }^{\mathrm{b}}$ Hadassah Academic College, 7 Hanevi'im Street, Jerusalem 9101001, Israel
}

Corresponding Author

*Dan T. Major (majort@biu.ac.il) 


\section{Content}

Figure S1. Optimally docked poses of substrate, intermediates, and product in (+)-bornyl diphosphate synthase (including a single active site crystal water). Docking was performed with Glide.

Figure S2. Optimally docked poses of substrate, intermediates, and product in (+)-bornyl diphosphate synthase (excluding active site crystal water). Docking was performed with Glide.

Figure S3. Overlay of all ligands (substrate, intermediates, and product) docked in (+)-bornyl diphosphate synthase. Docking was performed with Glide.

Figure S4. Optimally docked poses of substrate, intermediates, and product in the diterpene synthase CotB2. Docking was performed with Glide.

Figure S5. Overlay of all ligands (substrate, intermediates, and product) docked in the diterpene synthase CotB2. Docking was performed with Glide.

Figure S6. Optimally docked poses of substrate, intermediate, transition state, and product in the (Retro)Diels-Alderase LepI. Docking was performed with Glide.

Figure S7. Overlay of all ligands (substrate, intermediate, transition state, and product) docked in the (Retro)DielsAlderase LepI. Docking was performed with Glide.

Figure S8. Optimally docked poses of substrate, transition state, and product in the Diels-Alderase AbyU. Docking was performed with Glide.

Figure S9. Overlay of all ligands (substrate, transition state, and product) docked in the Diels-Alderase AbyU. Docking was performed with Glide.

Figure S10. Superimposition between docked pose from Enzydock and crystal structure of (+)-bornyl diphosphate synthase.

Figure S11. Superimposition between docked pose from Enzydock and crystal structure of (+)-bornyl diphosphate synthase. NOE restraints were imposed between ligand and diphosphate.

Figure S12. Superimposition between docked pose from Enzydock and crystal structure of (+)-bornyl diphosphate synthase. NOE restraints were imposed between ligand and diphosphate. Harmonic BESTFIT restraints were applied to the ligands. 
Figure S13. Superimposition between docked pose from Enzydock and crystal structure of (+)-bornyl diphosphate synthase. Crystal water was excluded from active site.

Figure S14. Overlay of all ligands (substrate, intermediates, and product) docked in (+)-bornyl diphosphate synthase using EnzyDock.

Figure S15. RMSD violin plots for all docked poses of the ligands in (+)-bornyl diphosphate synthase.

Figure S16. RMSD violin plots for all docked poses of the ligands in (+)-bornyl diphosphate synthase. NOE restraints were imposed between ligand and diphosphate

Figure S17. RMSD violin plots for all docked poses of the ligands in (+)-bornyl diphosphate synthase. NOE restraints were imposed between ligand and diphosphate. Harmonic BESTFIT restraints were applied to the ligands.

Figure S18. Superimposition between docking pose of Enzydock and crystal structure of the diterpene cyclase, CotB2.

Figure S19. Superimposition between docked pose from Enzydock and substrate bound crystal structure of the diterpene cyclase, CotB2.

Figure S20. Superimposition between docked pose from Enzydock and crystal structure of the diterpene cyclase, CotB2. NOE restraints were imposed between ligand and diphosphate.

Figure S21. Superimposition between docked pose from Enzydock and crystal structure of the diterpene cyclase, CotB2. NOE restraints were imposed between ligand and diphosphate. Harmonic BESTFIT restraints were applied to the ligands.

Figure S22. Overlay of all ligands (substrate, intermediates, and product) docked in the diterpene synthase, CotB2. Docking was performed with EnzyDock.

Figure S23. Superimposition of docked poses in the diterpene synthase, CotB2, (Grid, Full MM and QM/MM) obtained from EnzyDock.

Figure S24. RMSD violin plots for all docked poses of the ligands in the diterpene cyclase, CotB2.

Figure S25. RMSD violin plots for all docked poses of the ligands in the diterpene cyclase, CotB2. NOE restraints were imposed between ligand and diphosphate.

Figure S26. RMSD violin plots for all docked poses of the ligands in diterpene cyclase, CotB2. NOE restraints were imposed between ligand and diphosphate. Harmonic BESTFIT restraints were applied to the ligands.

Figure S27. Overlay of all ligands (substrate, intermediate, transitions states and products) docked in LepI. Docking was performed with EnzyDock. 
Figure S28. Overlay of docking poses of covalently bonded ligand in the cysteine protease cruzain. Docking was performed with EnzyDock.

Table S1. EnzyDock code runs with different crystal structures for BPPS.

Table S2. EnzyDock code runs with (+)-bornyl diphosphate synthase.

Table S3. EnzyDock code runs with (+)-bornyl diphosphate synthase. NOE restraints were imposed between ligand and diphosphate.

Table S4. EnzyDock code runs with (+)-bornyl diphosphate synthase. NOE restraints were imposed between ligand and diphosphate. Harmonic BESTFIT restraints were applied to the ligands.

Table S5. EnzyDock code runs with the diterpene cyclase, CotB2.

Table S6. EnzyDock code runs with the diterpene cyclase, CotB2. NOE restraints were imposed between ligand and diphosphate.

Table S7. EnzyDock code runs with the diterpene cyclase, CotB2. NOE restraints were imposed between ligand and diphosphate. Harmonic BESTFIT restraints were applied to the ligands.

Table S8. Geometric changes in active site for Michaelis complexes and transition state in proline racemase.

Table S9. RMSD ( $\AA$ ) values of the five best EnzyDock docked poses relative to the crystal structure of cruzain. 


\section{Ligand Docking with Glide}

To compare the performance of EnzyDock to a well-established docking program, we performed ligand docking with Glide ${ }^{1-3}$ for all systems (i.e. (+)-bornyl diphosphate synthase, ${ }^{4}$ the bacterial diterpene synthase CotB2, ${ }^{5}$ spirotetronate $\mathrm{AbyU},{ }^{6} \mathrm{LepI}^{7}$ and the cysteine protease cruzain ${ }^{8-9}$. We performed three basic steps in flexible ligand docking with Glide: (1) preparation of the protein and ligand structures, (2) grid generation, and (3) flexible ligand docking. Prior to docking with Glide, protein and ligands were prepared using Schrödinger's Maestro (version 11.4, 2017-4 release) Prep $\mathrm{Wiz}^{10}$ and LigPrep module, ${ }^{11}$ respectively, with default settings. Using the Prep Wiz module, all crystal water molecules were deleted, missing side chains were added (where needed), $\mathrm{pK}_{\mathrm{a}}$ values were calculated to assign the correct protonation state for all titratable residues $($ at $\mathrm{pH}=7.0)$. We carefully checked the protonation state of each titratable residues to generate the exact receptor, as used with the EnzyDock docking program (which were prepared using CHARMM). Using the LigPrep module, protonation states were calculated at $\mathrm{pH}=7.0$ and specific chiralities were retained. Ligands with correct stereochemistry were selected for flexible ligand docking and the stereochemistry was preserved during docking. To ensure that the correct ligand structure is used, each substrate, intermediate, transition state and product were manually inspected.

Grid generation. To allow Glide to search for favorable interactions between the ligand and binding-site region, we generated the grid to represent the protein and cofactors (if present). Grid represents the shape and properties of the receptor and is used for positioning and scoring the ligand poses. The grid location is set based on the crystallographic ligand position. The centroid of the input ligand is used to generate the grid center. Glide does not allow for docking with receptor flexibility but reducing van der Waals $(\mathrm{vdW})$ radii of nonpolar atoms can mimic the effect of receptor flexibility to a certain degree. For all systems studied in this manuscript, vdW radii of nonpolar receptor atoms are specified by a scaling factor of 1.0 and the partial charge cutoff is set to 0.25 (default value). The scaling of vdW radii is performed only for nonpolar atoms, defined as those for which the absolute value of the partial atomic charge is less than or equal to 0.25 . Glide uses two grid boxes to perform the docking calculation. The enclosing box defines the space in which grids are calculated. This is also the box within which all the ligand atoms must be contained. The bounding box defines the space within which the ligand center must be contained. The ligand center is defined as the midpoint of the line drawn between the two most distal atoms. Both boxes share a common center, which is set as the centroid of the input ligand. The dimensions of the enclosing box and the bounding box are set to $10 \AA$ and $20 \AA$ (defaults values), respectively, for all ten systems. We note that in the case of bornyl diphosphate synthase, to investigate the effect of a single crystal water in the active site, we generated two different types of grids: (1) grid with one crystal water in the active site (See Fig. S1) and (2) grid without any water (See Fig. S2). The other cofactors (diphosphate and 3 magnesium ions) remain the same for both cases.

Flexible Ligand docking. Glide performs flexible ligand docking into a rigid protein environment. ${ }^{12}$ There are two primary goals of flexible ligand docking: to accurately predict ligand poses and to rank ligands by predicted binding affinities to the protein. The number of poses written per ligand is set to at most 100 poses. To soften the potential for 
the nonpolar parts of the ligands, the scaling factor of the vdW radii is set to the default value of 0.80 , while the partial atomic charge cutoff is set to 0.15 by default. Standard precision (SP) mode is chosen for most ligand docking runs. ${ }^{1}$ For the Diels-Alderase AbyU, Extra precision (XP) mode was considered, as SP mode was unable to produce any chemically relevant poses in the active site of the receptor. XP mode commences with SP sampling before continuing with an anchor-and-grow procedure for ligand sampling. XP further employs a more sophisticated scoring function that is "harder" than the SP GlideScore, with greater requirements for ligand-receptor shape complementarity. During the docking process, conformations are generated by setting the energy window for ring sampling to $2.5 \mathrm{kcal} / \mathrm{mol}$ (default). The "Canonicalize input conformation" option is deactivated. Ligand poses are filtered out by Coulomb and vdW interaction energies greater than $0 \mathrm{kcal} / \mathrm{mol}$ for BPPS, CotB2, and LepI, $700 \mathrm{kcal} / \mathrm{mol}$ for reactant and product in AbyU, and $1000 \mathrm{kcal} / \mathrm{mol}$ for transition state in AbyU. Post docking minimization of all the docked poses are performed with 100 minimization steps, and a distance-dependent dielectric constant with a value of 2.0 is used to account electrostatic interactions. During the final stage, all poses are rescored using GlideScore. The selection of the best-docked structure for a ligand among the energy minimized poses is made using a model energy score called "Emodel" that combines the energy score, the binding affinity predicted by GlideScore, and the internal strain energy for the model potential used to direct the conformation generation algorithm.

Rigid docking of transition state structure. In our study, transition states of the ligands are generated by gas phase QM calculations. ${ }^{7}$ The gas phase calculations were performed within the framework of DFT using the Gaussian 16 software package. ${ }^{13}$ The specific flavor of DFT is the B97 functional with Grimme's dispersion correction, i.e. B97D, ${ }^{14-15}$ in conjunction with the $6-31+\mathrm{G}(\mathrm{d}, \mathrm{p})$ basis set. ${ }^{16-17}$ The transition state conformation is docked rigidly into the protein binding site with Glide (ligand sampling: rigid). In this mode, the ligand is translated and rotated relative to the binding site, but no conformational sampling is performed for the ligand during the docking step. One pose is saved for each docked conformation.

Covalent docking. We performed covalent docking in Cysteine protease Cruzain, hepatitis C virus NS5b RNA polymerase, tyrosine kinase, and adipocyte fatty acid binding protein using the CovDock module available in Glide. ${ }^{8}$ All the default settings of this module were used throughout the study.

In all Glide docking calculations, the OPLS3 force field is used for protein preparation, ligand preparation, conformational analysis, Glide Grid generation, and docking studies. ${ }^{18}$ All figures were created utilizing the PyMOL software. ${ }^{19}$ 


\section{Glide Docking Results}

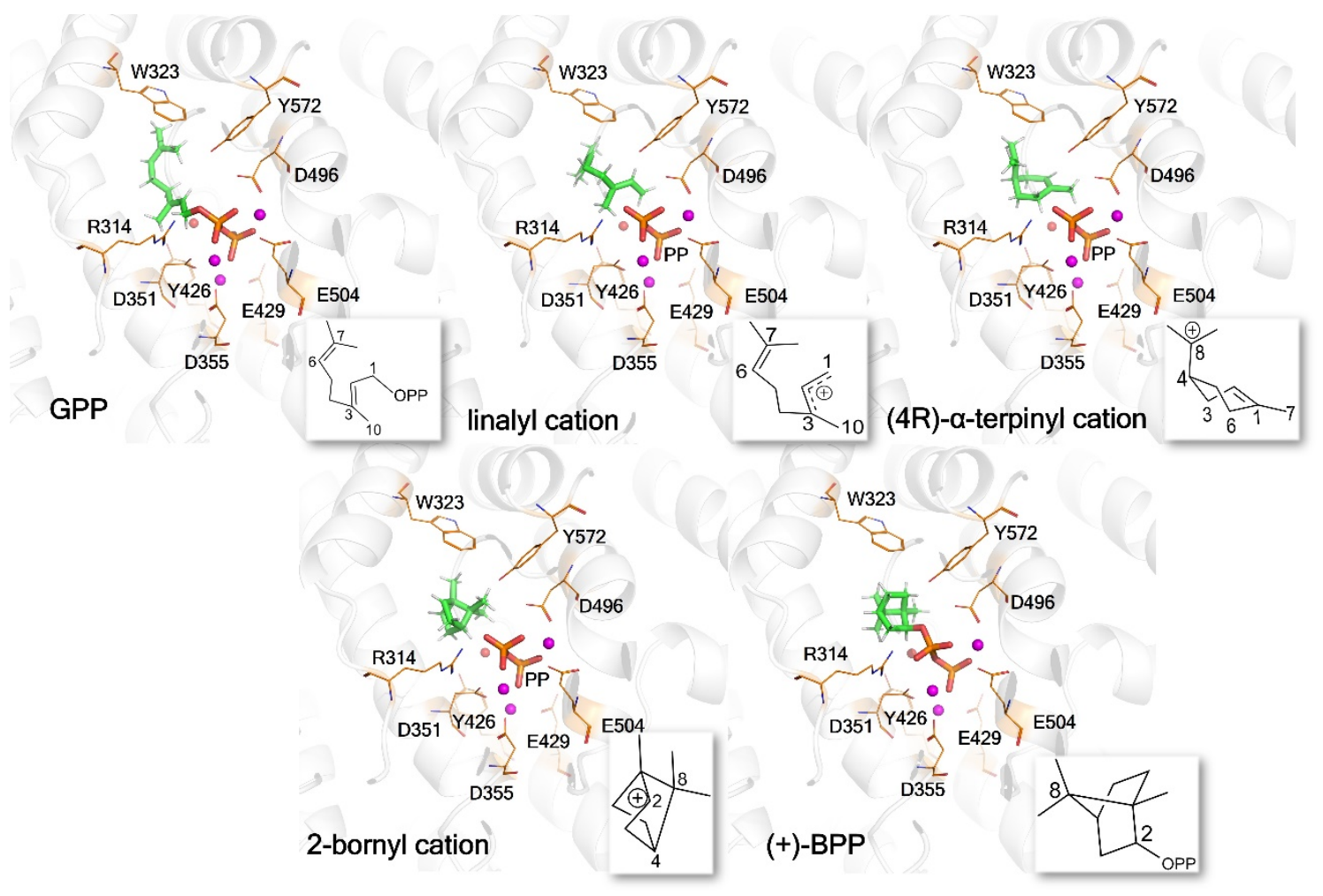

Figure S1. Glide docked poses of substrate, intermediates, and product in (+)-bornyl diphosphate synthase. The red sphere represents an active site crystal water molecule and magenta spheres represent $\mathrm{Mg}^{2+}$ ions. Ligand carbons are shown in green. 


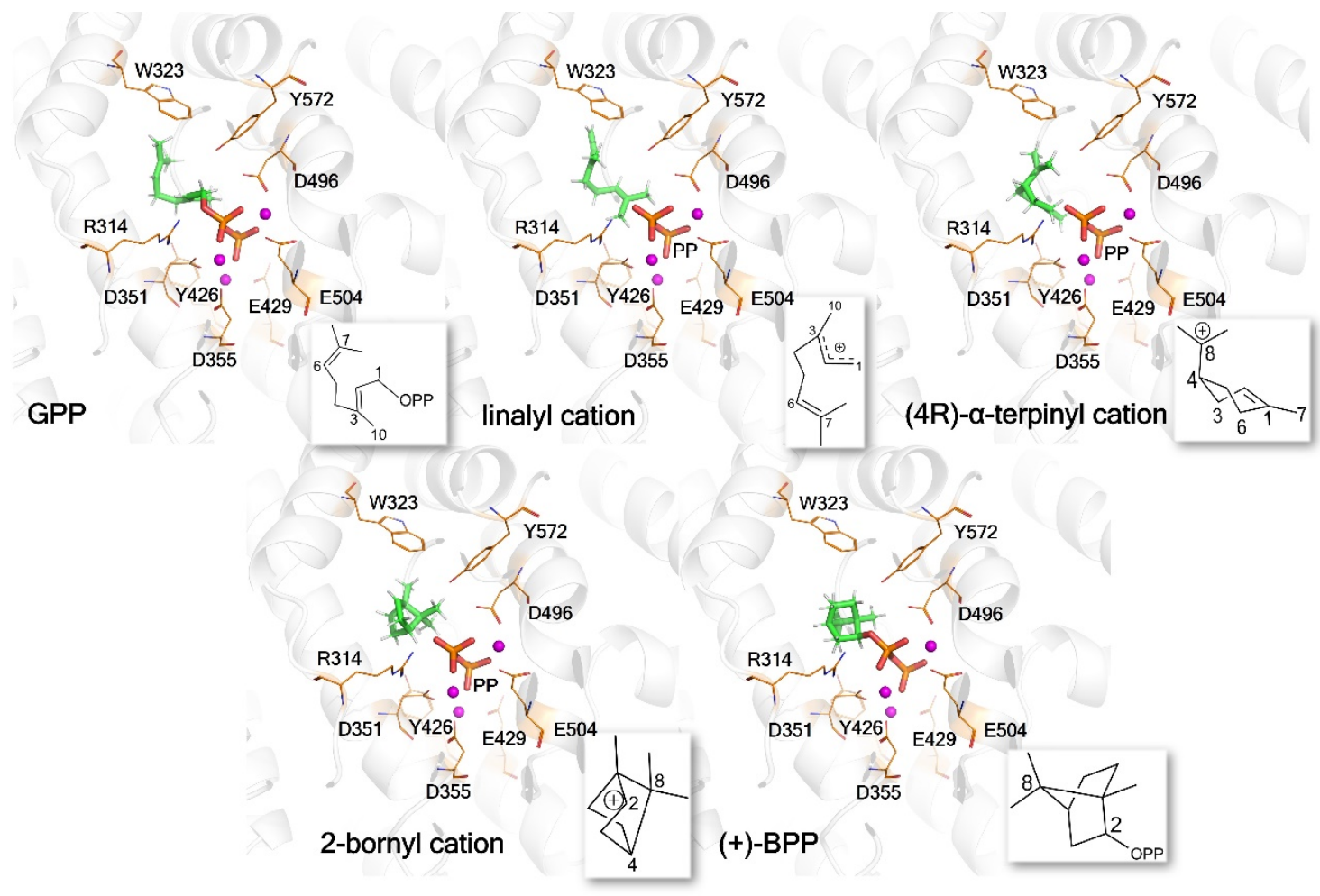

Figure S2. Glide docked poses of substrate, intermediates, and product in (+)-bornyl diphosphate synthase (excluding active site crystal water). Magenta spheres represent $\mathrm{Mg}^{2+}$ ions. Ligand carbons are shown in green. 


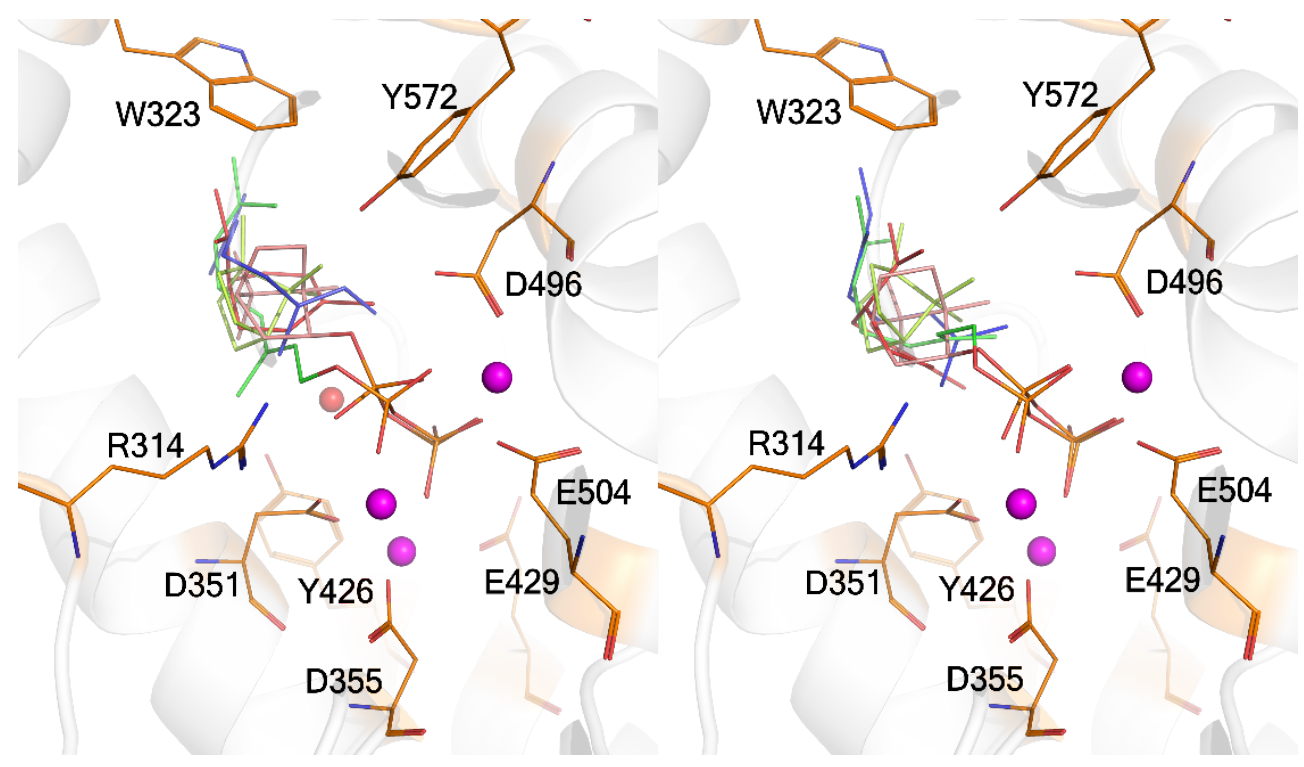

Figure S3. Overlay of all ligands (substrate, intermediates, and product) docked in (+)-bornyl diphosphate synthase using Glide. Geranyl diphosphate, linalyl cation, (4R)- $\alpha$-terpinyl cation, 2-bornyl cation and (+)-bornyl diphosphate are represented by green, blue, red, lemon and salmon color, respectively. Magenta spheres represent $\mathrm{Mg}^{2+}$ ions. Left: Glide docking including an active site water molecule (red sphere). Right: Glide docking excluding active site water. 


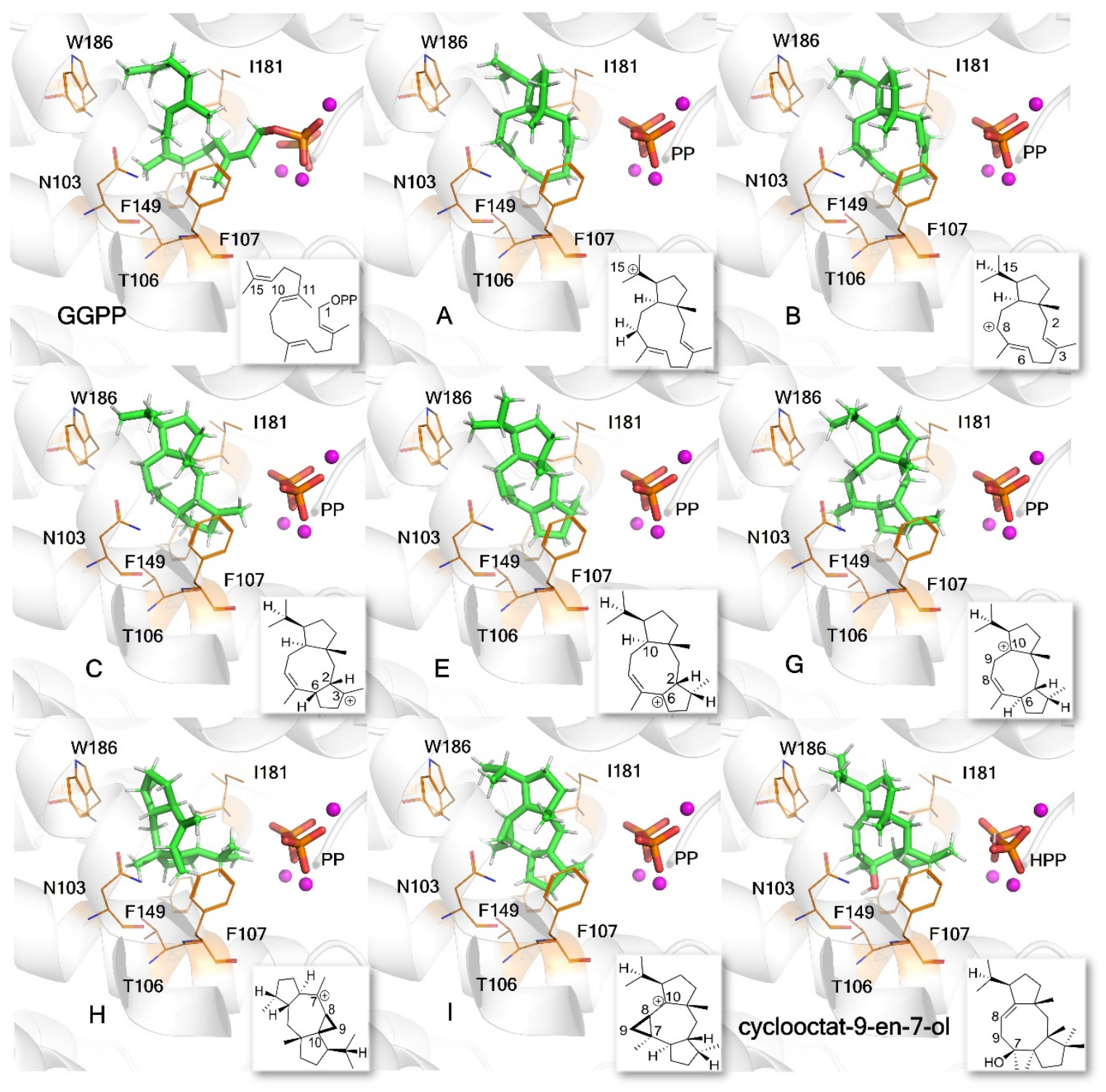

Figure S4. Glide docked poses of substrate, intermediates, and product in the diterpene synthase, CotB2. Magenta spheres represent three $\mathrm{Mg}^{2+}$ ions. Ligand carbons are shown in green. 


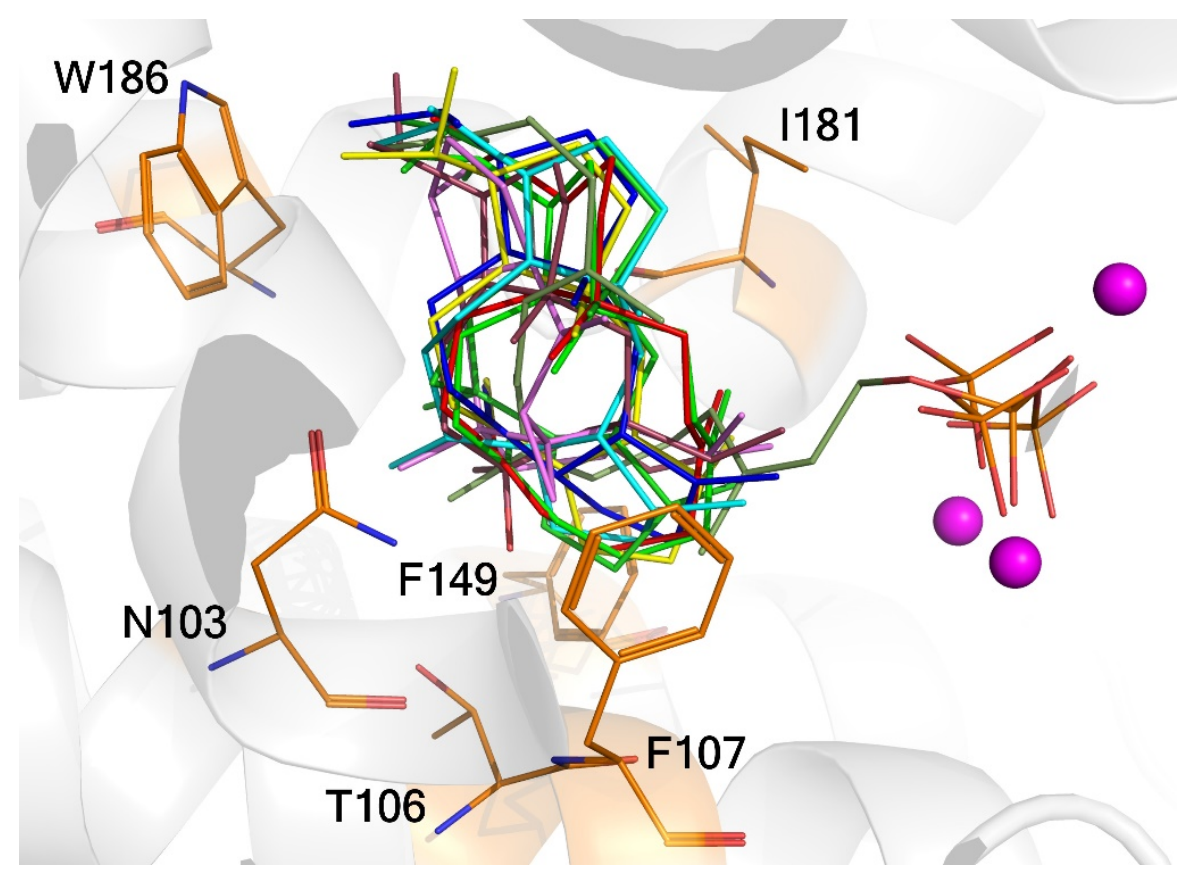

Figure S5. Overlay of all ligands (substrate, intermediates, and product) docked in the diterpene synthase, CotB2, using Glide. Geranyl geranyl diphosphate, intermediates viz. A, B, C, E, G, H, I and product cyclooctat-9-en-7-ol are represented by forest, red, green, blue, yellow, cyan, violet, deep-olive and dirty-violet color, respectively. Magenta spheres represent $\mathrm{Mg}^{2+}$ ions. 


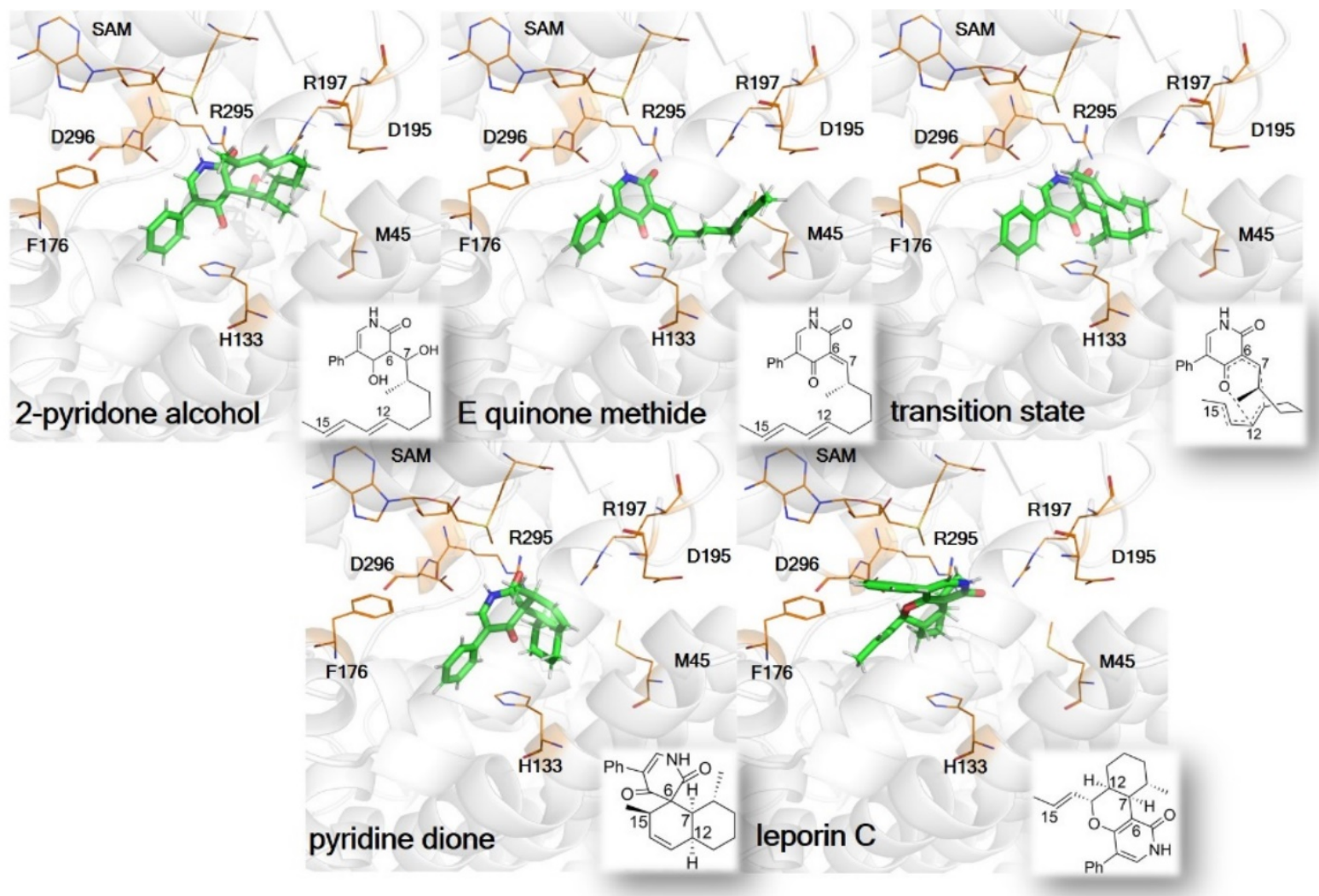

Figure S6. Docked poses of substrate, intermediate, transitions state, and products in LepI using Glide. Ligand carbons are shown in green. 


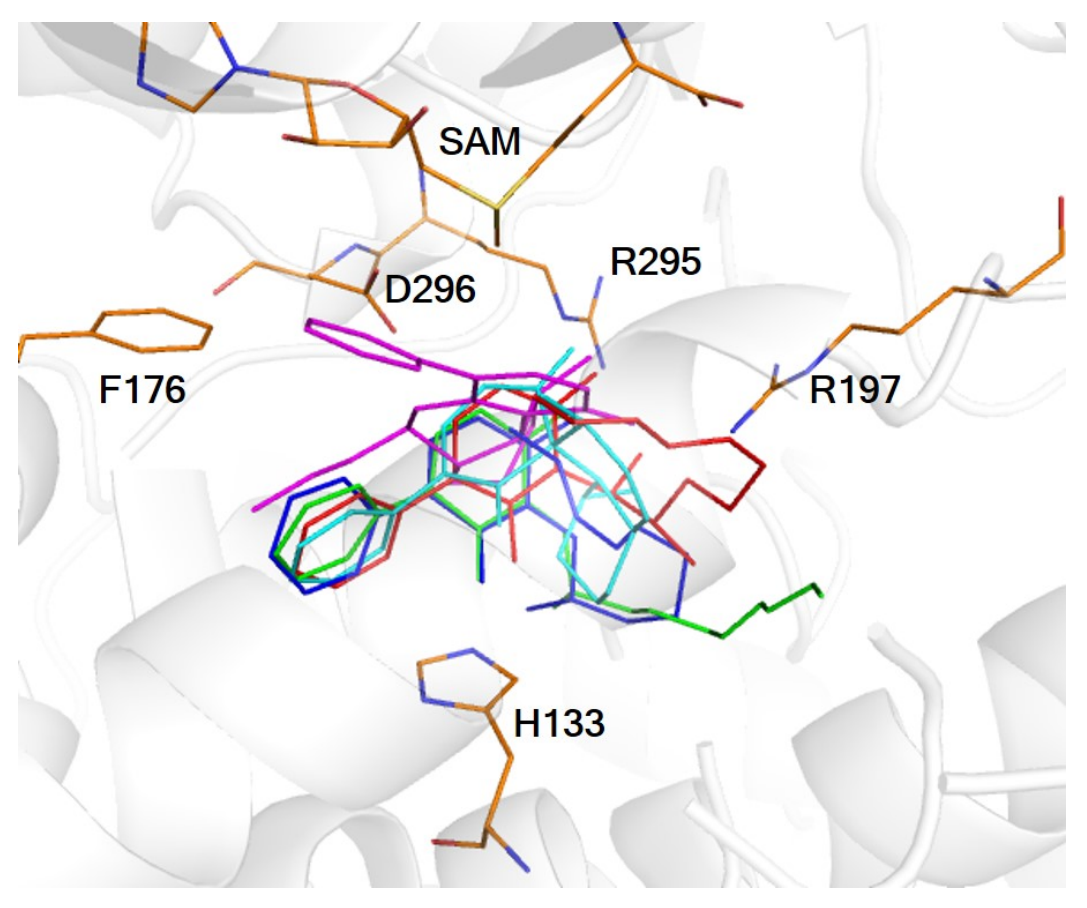

Figure S7. Overlay of all ligands (substrate, intermediate, transitions states and products) docked in LepI using Glide. Substrate (2-pyridone alcohol), intermediate (E quinone methide), transition state, and products (leporin C, pyridine dione) are represented by red, green, blue, cyan and magenta colors, respectively. 

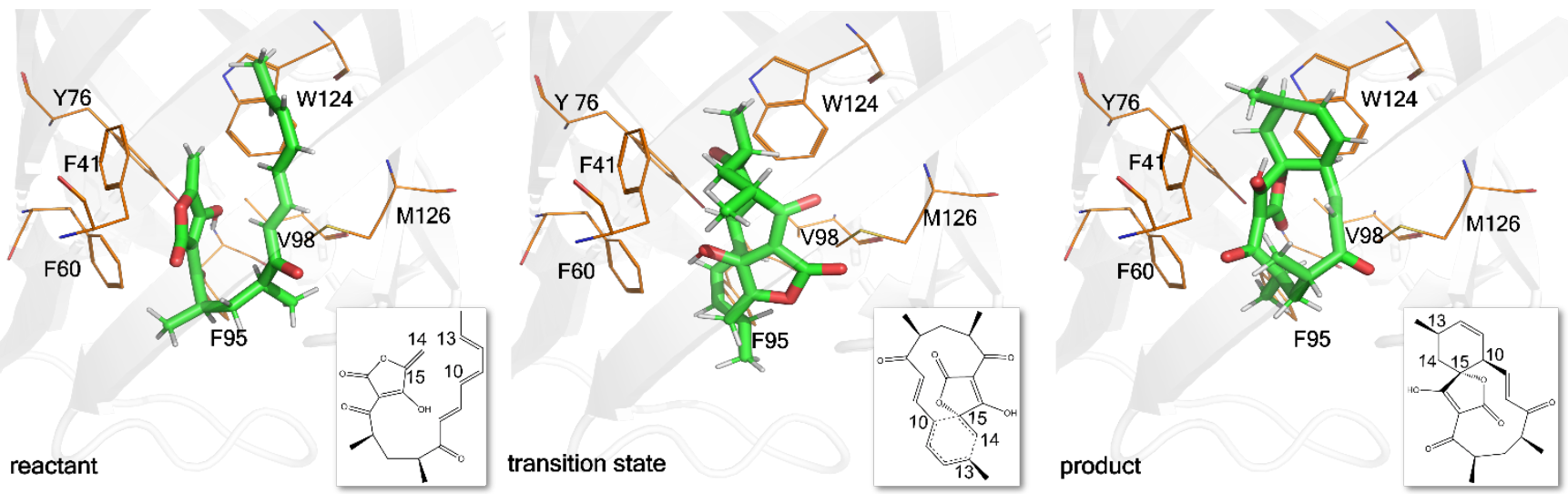

Figure S8. Docked poses of substrate, transition state, and product in the Diels-Alderase, AbyU, using Glide. Ligand carbons are shown in green. 


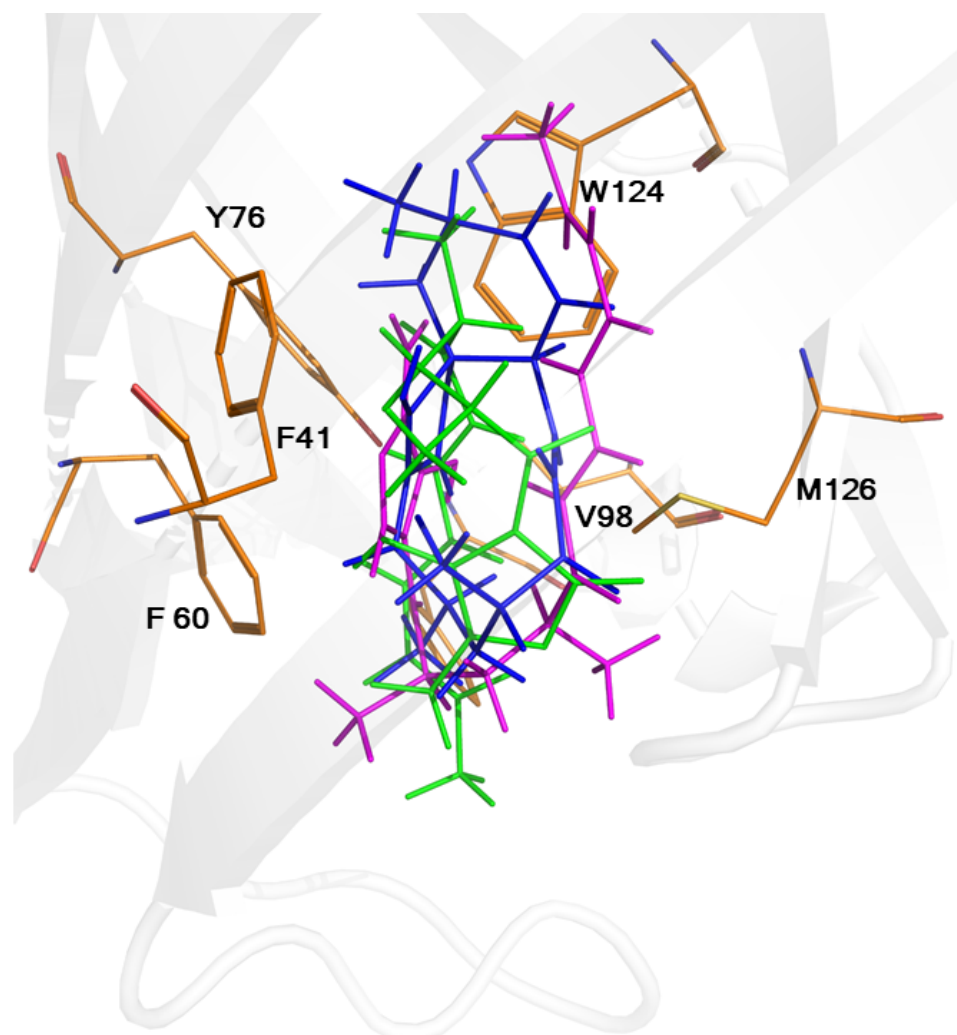

Figure S9. Overlay of substrate, transition state, and product docked in the Diels-Alderase, AbyU, using Glide. Substrate, transition state, and product are represented by magenta, green and blue, respectively. 


\section{EnzyDock Results (BPPS)}

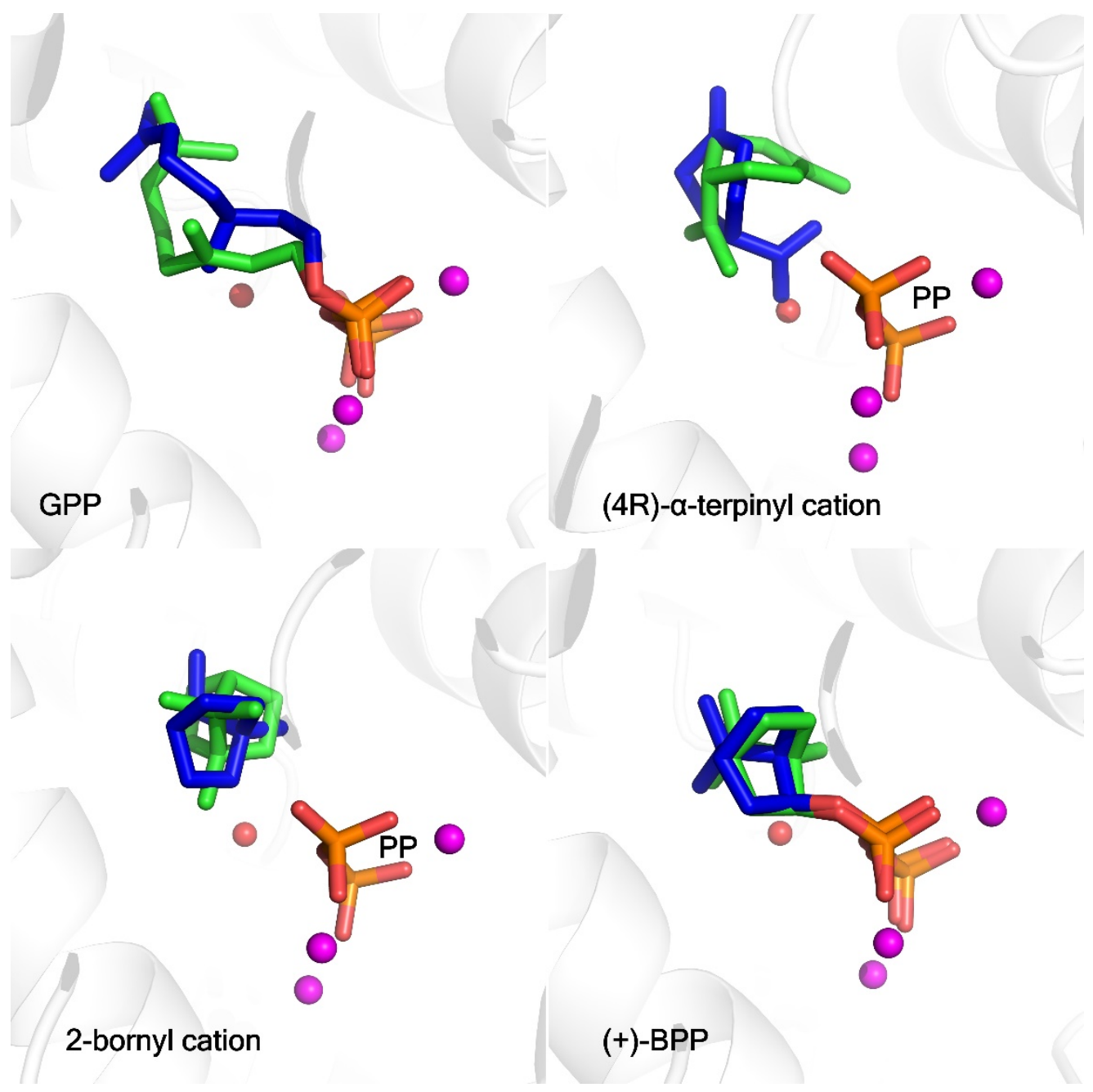

Figure S10. Overlay of poses docked with EnzyDock and the crystal structure of (+)-bornyl diphosphate synthase. ${ }^{20}$ Green and blue represent docked poses and crystal structure, respectively. The red sphere represents a crystal water molecule in the active site and magenta spheres represent three $\mathrm{Mg}^{2+}$ ions. 


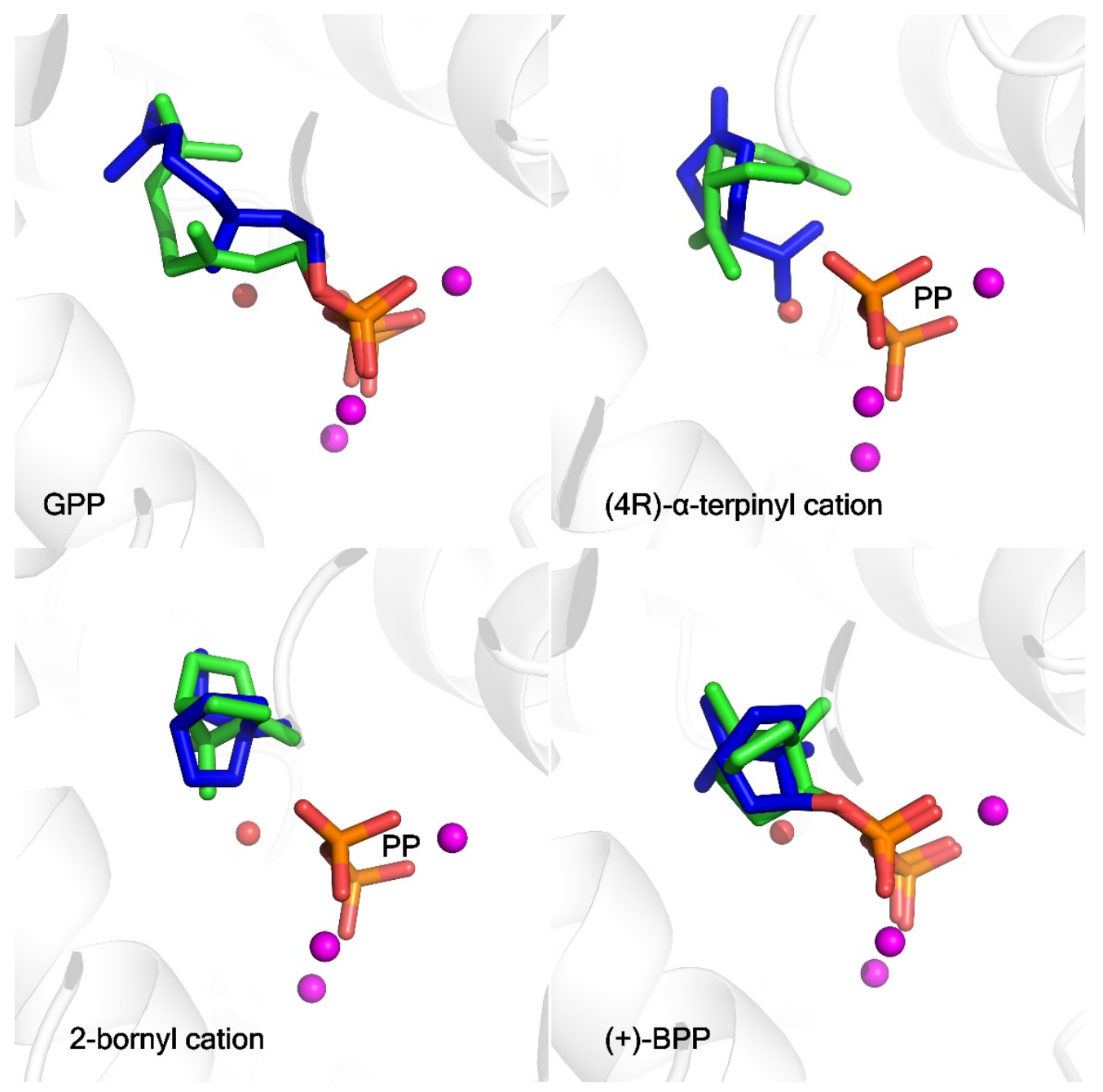

Figure S11. Overlay of poses docked with EnzyDock and the crystal structure of (+)-bornyl diphosphate synthase. ${ }^{20}$ NOE restraints are imposed between ligand $\mathrm{C} 1 / \mathrm{C} 2$ carbon atoms and $\mathrm{O} 1$ oxygen atom of the diphosphate, and between diphosphate and $\mathrm{Mg}^{2+}$ ions. Green and blue colors represent docked poses and crystal structure, respectively. The red sphere represents a crystal water molecule in the active site and magenta spheres represent three $\mathrm{Mg}^{2+}$ ions. 


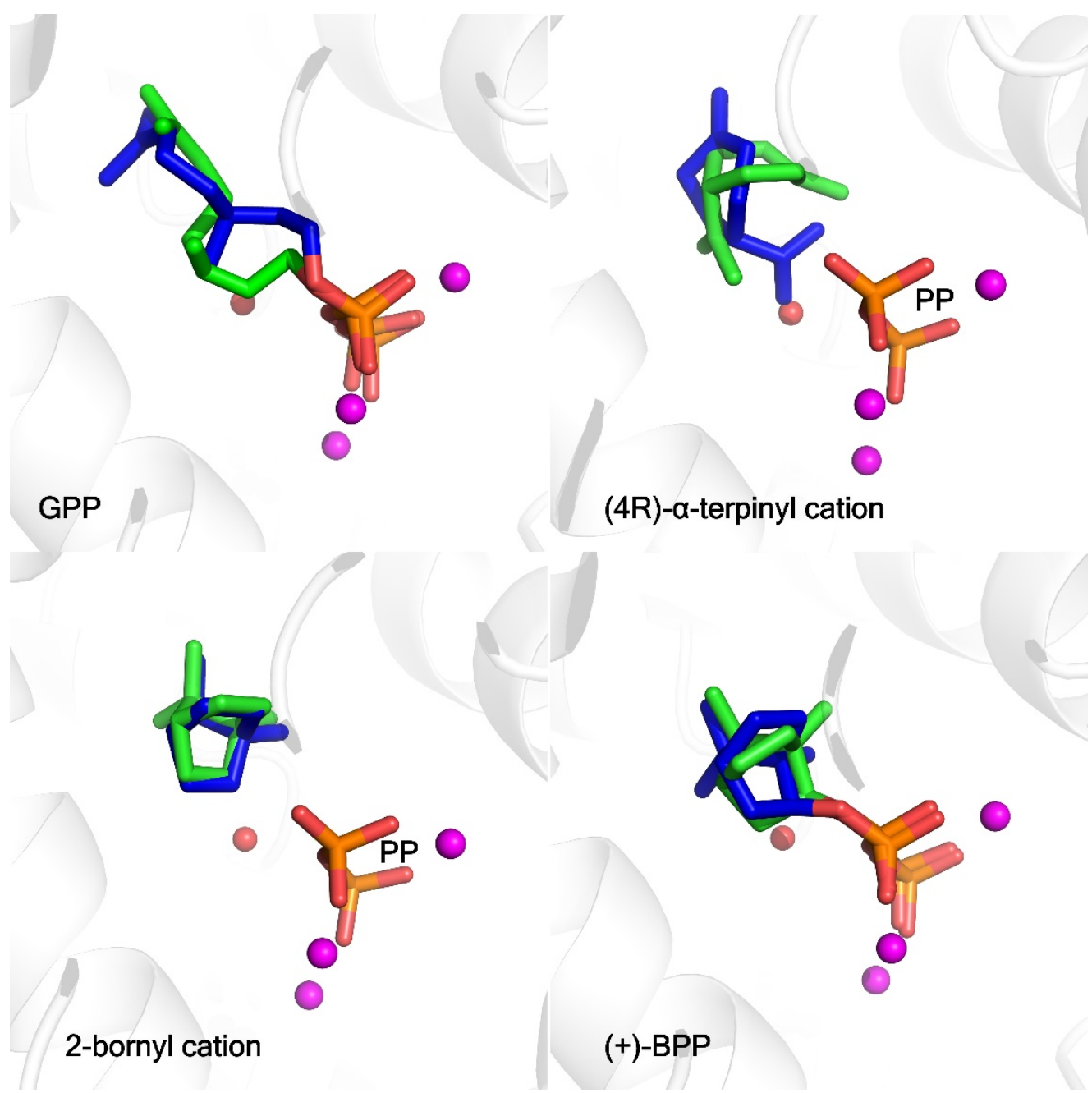

Figure S12. Overlay of poses docked with EnzyDock and the crystal structure of (+)-bornyl diphosphate synthase. NOE restraints are imposed between ligand $\mathrm{C} 1 / \mathrm{C} 2$ carbon atoms and $\mathrm{O} 1$ oxygen atom of diphosphate, and between diphosphate and $\mathrm{Mg}^{2+}$ ions. Harmonic BESTFIT restraints are applied to several carbon atoms of the ligands. Green and blue colors represent docked poses and crystal structure, respectively. The red sphere represents a crystal water molecule in the active site and magenta spheres represent three $\mathrm{Mg}^{2+}$ ions. 

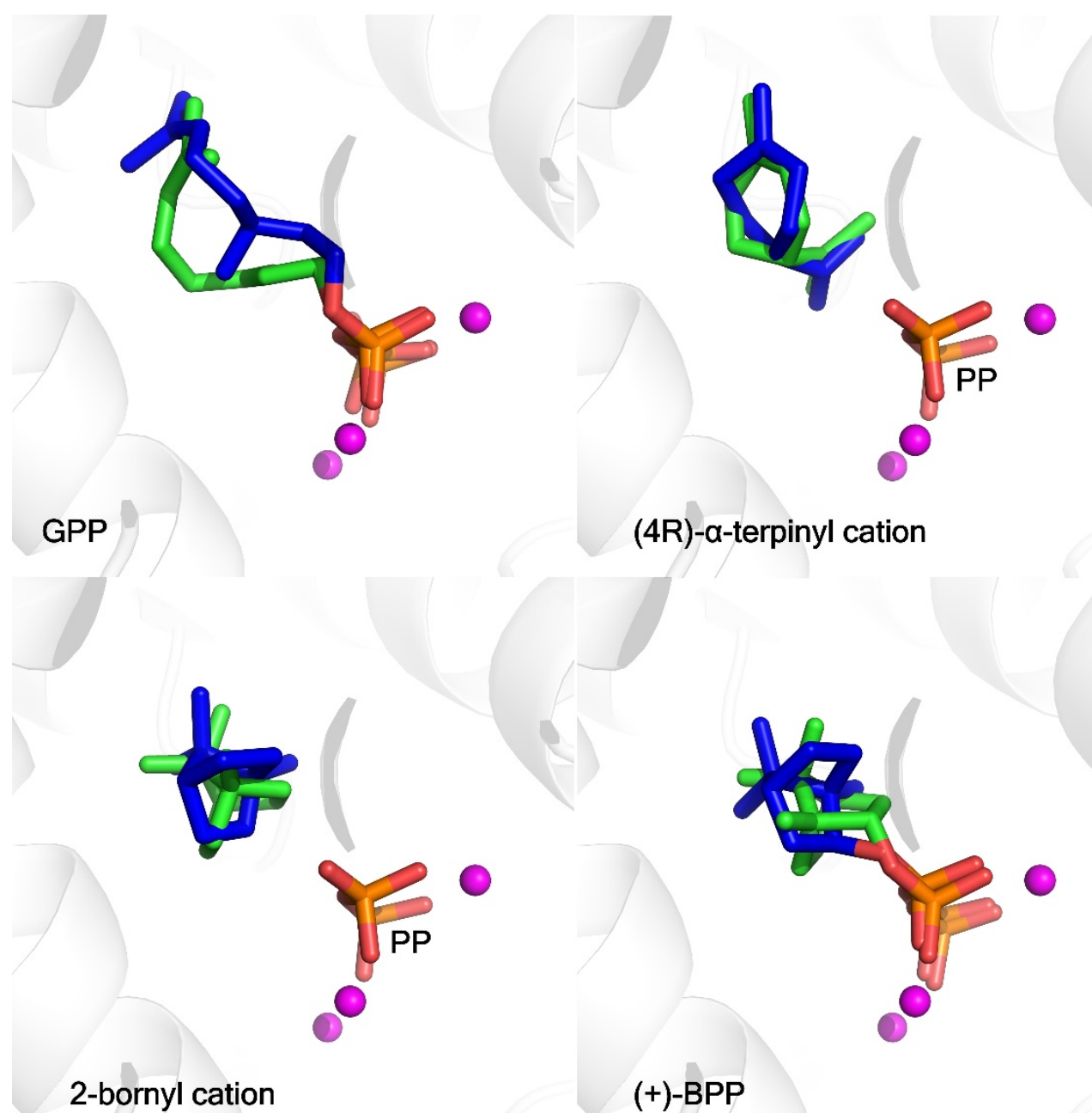

(4R)- $\alpha$-terpinyl cation

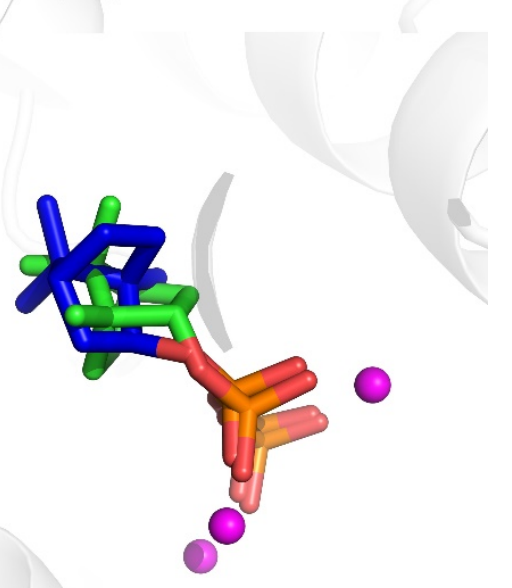

(+)-BPP

Figure S13. Overlay of poses docked with EnzyDock and crystal structure of (+)-bornyl diphosphate synthase. Docking was performed in the absence of the active site crystal water and no restraints were imposed. Green and blue colors represent docked poses and crystal structure, respectively. Magenta spheres represent three $\mathrm{Mg}^{2+}$ ions. 


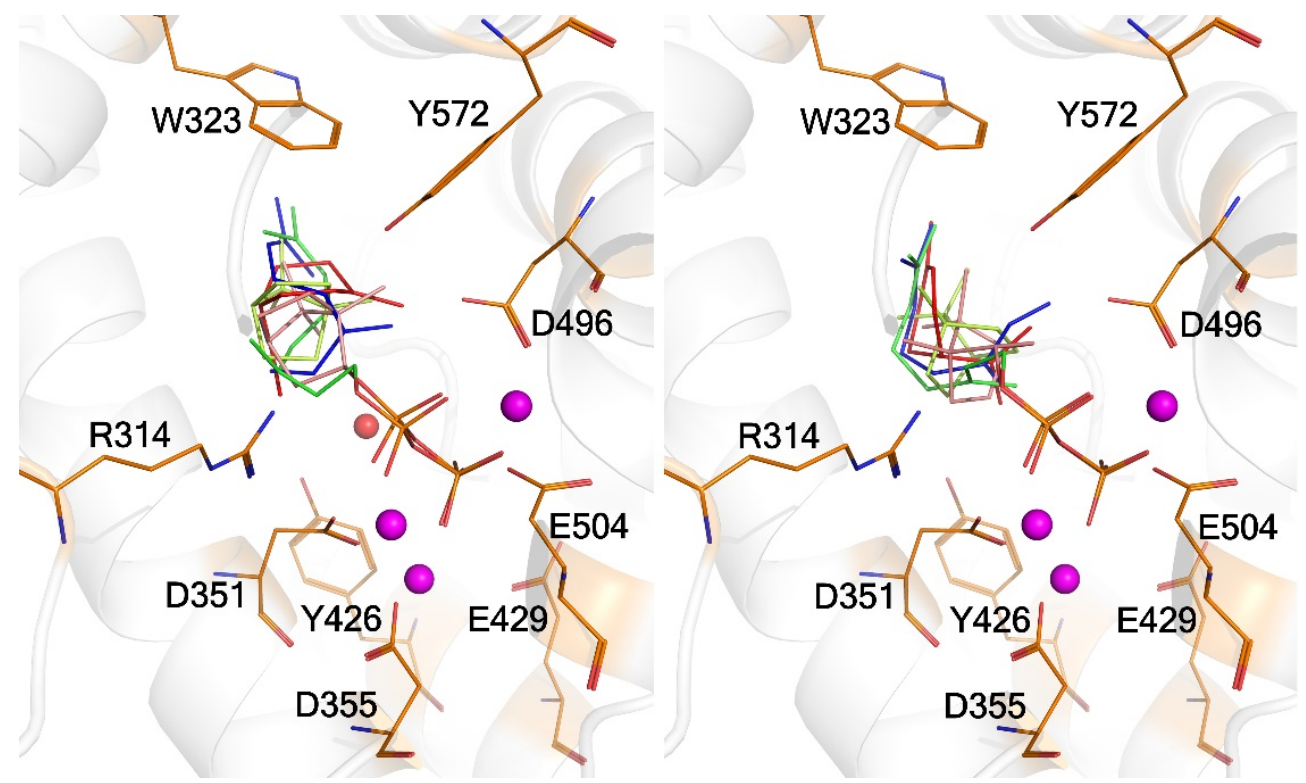

Figure S14. Overlay of all ligands (substrate, intermediates, and product) docked in (+)-bornyl diphosphate synthase using EnzyDock. Geranyl diphosphate, linalyl cation, (4R)- $\alpha$-terpinyl cation, 2-bornyl cation and (+)-bornyl diphosphate are represented by green, blue, red, lemon and salmon color, respectively. Magenta spheres represent $\mathrm{Mg}^{2+}$ ions. Left: EnzyDock restrained docking (NOE and BESTFIT harmonic force) including an active site water molecule (red sphere). Right: EnzyDock free docking excluding active site water. 


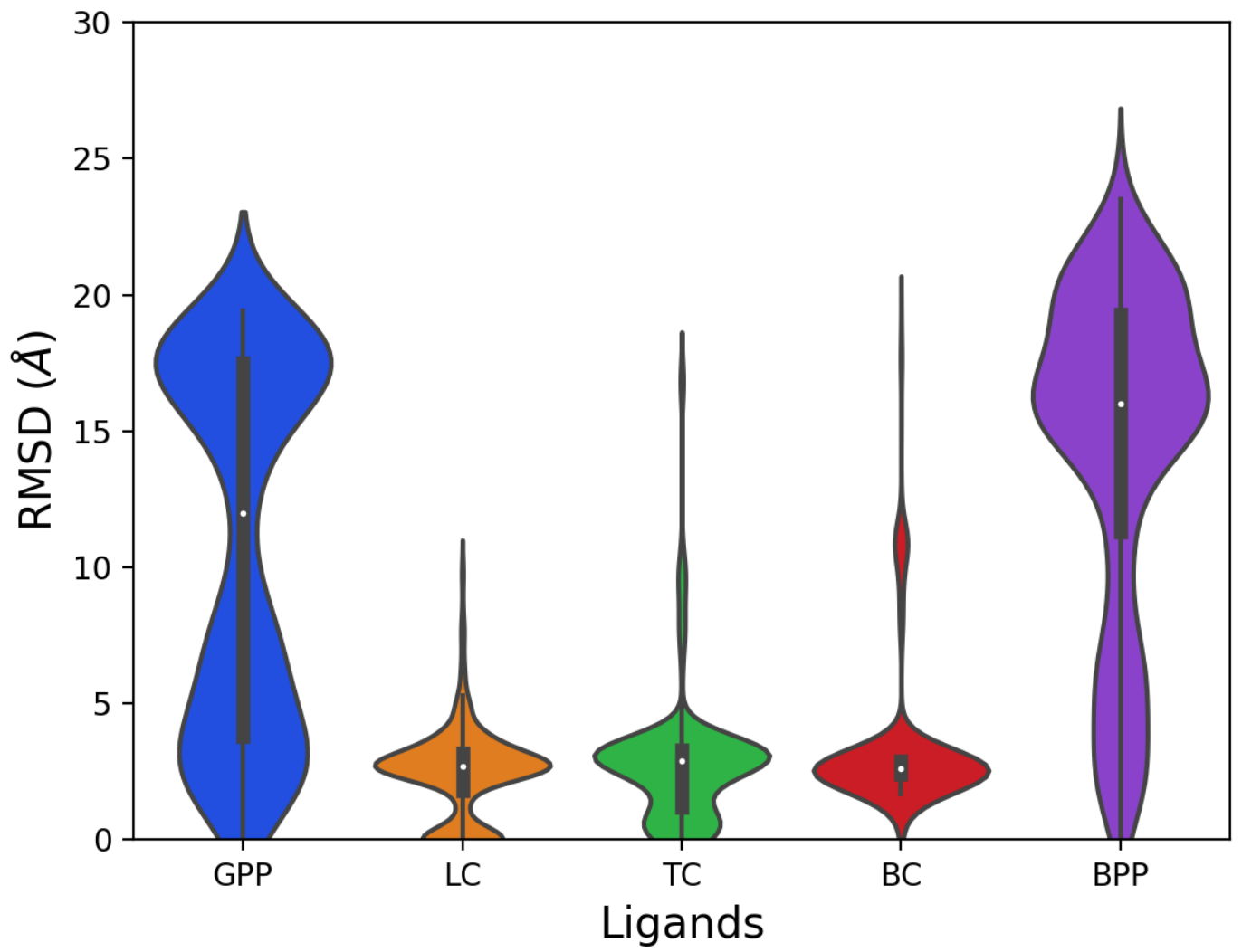

Figure S15. Violin plot of RMSD for all docked poses of the ligands in (+)-bornyl diphosphate synthase relative to the best docked pose (i.e. lowest EnzyDock energy). A total of 625 docked poses for each ligand are considered. A single crystal water, diphosphate and three $\mathrm{Mg}^{2+}$ ions are included as cofactors. 


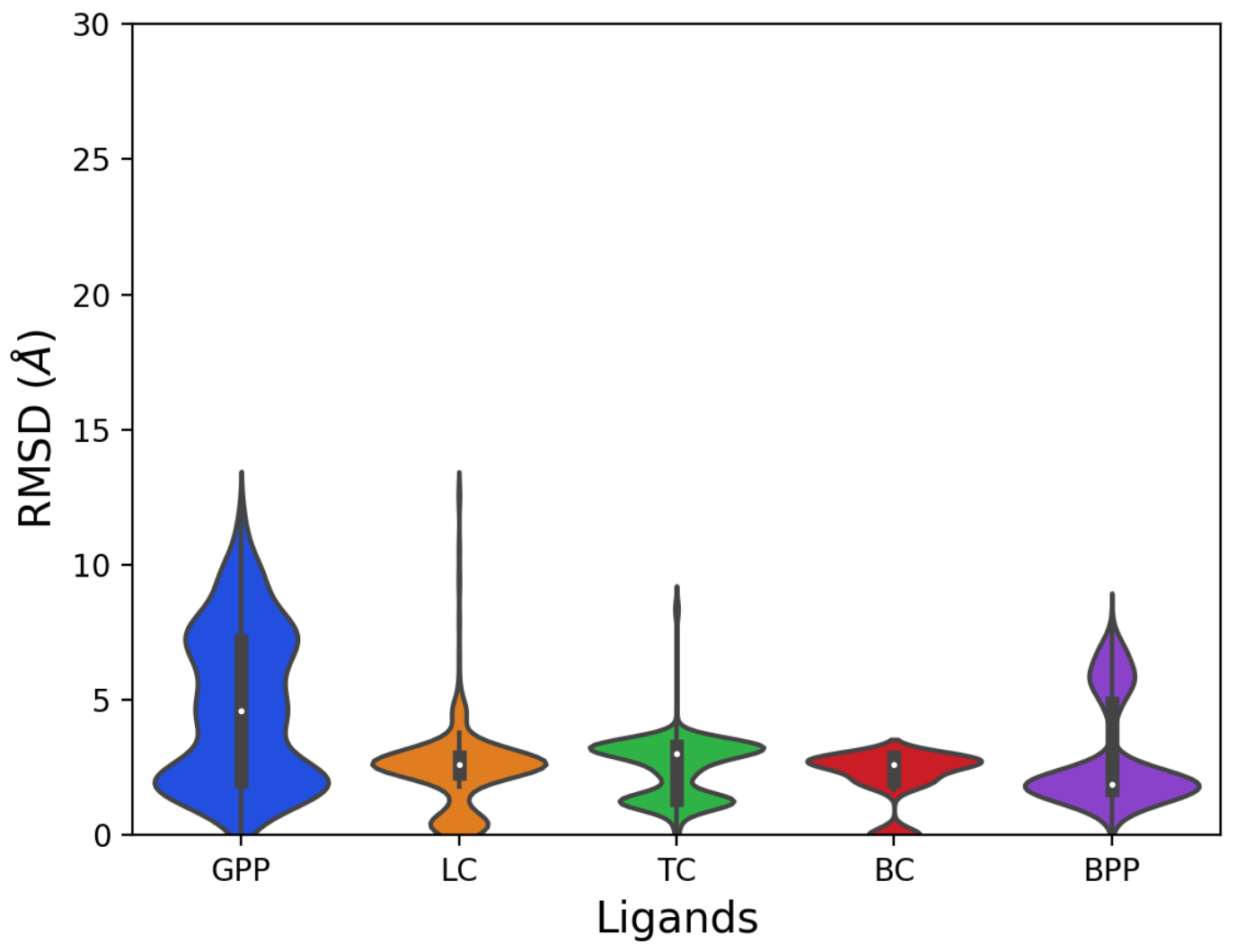

Figure S16. Violin plot of RMSD for all docked poses of the ligands in (+)-bornyl diphosphate synthase relative to the best docked pose (i.e. lowest EnzyDock energy). A total of 625 docked poses for each ligand are considered. A single crystal water, diphosphate and three $\mathrm{Mg}^{2+}$ ions are included as cofactors. NOE restrains are imposed between ligand $\mathrm{C} 1 / \mathrm{C} 2$ carbon atoms and $\mathrm{O} 1$ oxygen atom of the diphosphate, and between diphosphate and $\mathrm{Mg}^{2+}$ ions. 


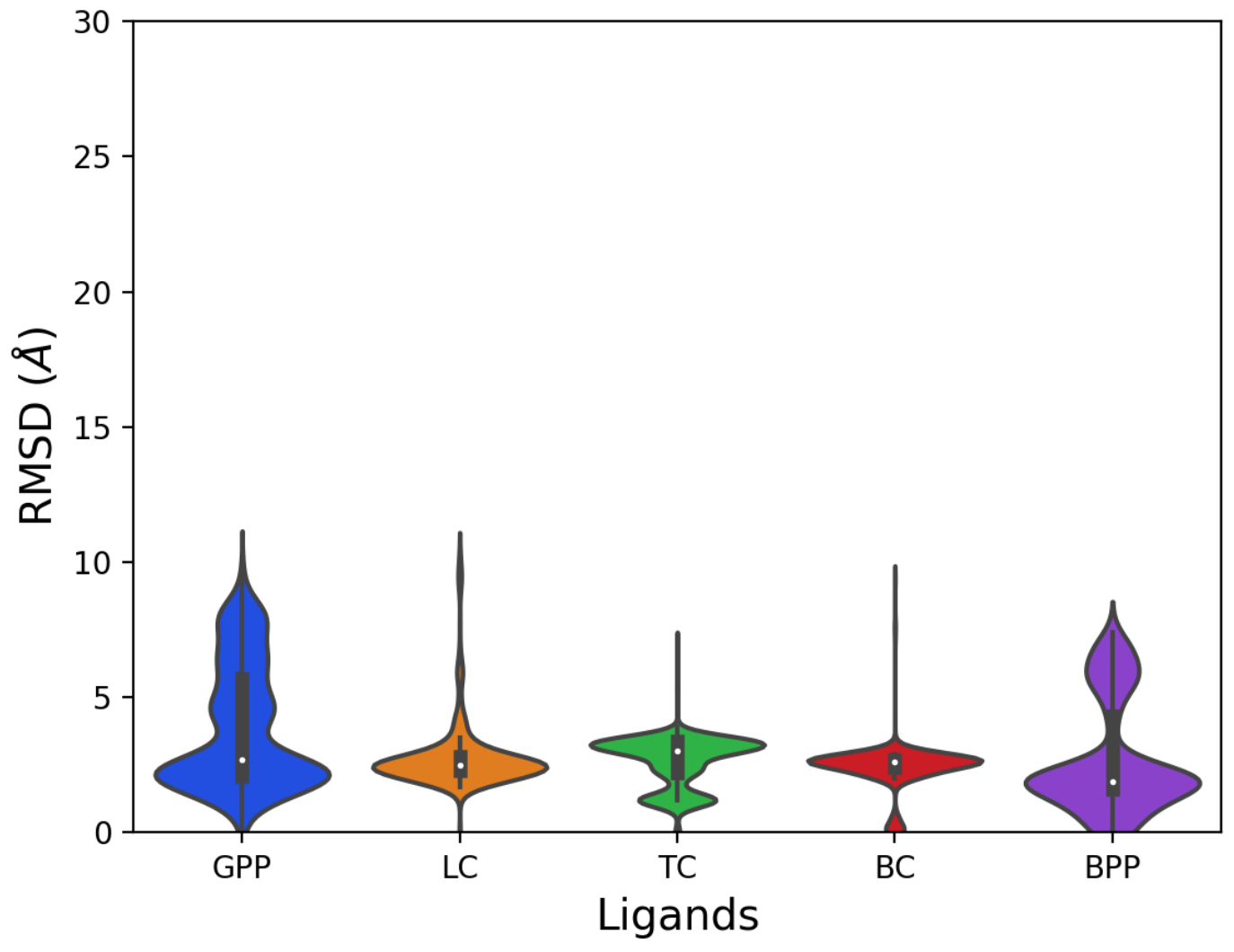

Figure S17. Violin plot of RMSD for all docked poses of the ligands in (+)-bornyl diphosphate synthase relative to the best docked pose (i.e. lowest EnzyDock energy). A total of 625 docked poses for each ligand are considered. A single crystal water, diphosphate and three $\mathrm{Mg}^{2+}$ ions are included as cofactors. NOE restrains are imposed between ligand $\mathrm{C} 1 / \mathrm{C} 2$ carbon atoms and $\mathrm{O} 1$ oxygen atom of diphosphate, and between diphosphate and $\mathrm{Mg}^{2+}$ ions. Harmonic BESTFIT restraints are applied to several carbon atoms of the ligands. 
Table S1. EnzyDock docking RMSD $(\AA ̊)$ values of substrate, intermediates and product using different BPPS crystal structures. The docking simulations employed crystal structures with PDB codes 1 N23 (holoenzyme where ligand is 2bornyl cation mimic (1R, 4S)-2-azabornane) and 1N24 (holoenzyme where ligand is the product (+)-BPP).

\begin{tabular}{|c|c|c|c|c|c|c|}
\hline \multirow[t]{3}{*}{ Ligands } & \multicolumn{2}{|c|}{ Free docking } & \multicolumn{4}{|c|}{ Restrained docking } \\
\hline & \multirow[b]{2}{*}{$1 \mathrm{~N} 23$} & \multirow[b]{2}{*}{$1 \mathrm{~N} 24$} & \multicolumn{2}{|c|}{ NOE } & \multicolumn{2}{|c|}{ NOE+BESTFIT } \\
\hline & & & $1 \mathrm{~N} 23$ & $1 \mathrm{~N} 24$ & $1 \mathrm{~N} 23$ & $1 \mathrm{~N} 24$ \\
\hline GPP & 1.7 & 2.0 & 1.7 & 1.7 & 1.5 & 1.7 \\
\hline (4R)- $\alpha$-terpinyl cation & 2.7 & 1.7 & 2.7 & 2.7 & 2.6 & 2.7 \\
\hline 2-bornyl cation & 3.0 & 2.0 & 2.0 & 2.0 & 0.4 & 0.6 \\
\hline$(+)-\mathrm{BPP}$ & 0.4 & 0.9 & 1.2 & 0.9 & 1.2 & 0.9 \\
\hline
\end{tabular}

Table S2. EnzyDock runs for (+)-bornyl diphosphate synthase. A single crystal water, diphosphate and three $\mathrm{Mg}^{2+}$ ions are included as cofactors. The consensus RMSD $(\AA)$ is calculated relative to the ligand, (+)-bornyl diphosphate.

\begin{tabular}{cccccc}
\hline EnzyDock Run No. & $\begin{array}{c}\text { geranyl } \\
\text { diphosphate }\end{array}$ & $\begin{array}{c}\text { linalyl } \\
\text { cation }\end{array}$ & $\begin{array}{c}(4 \mathrm{R}) \text { - } \alpha \text {-terpinyl } \\
\text { cation }\end{array}$ & $\begin{array}{c}\text { 2-bornyl } \\
\text { cation }\end{array}$ & $\begin{array}{c}(+) \text {-bornyl } \\
\text { diphosphate }\end{array}$ \\
\hline 1 & 2.4 & 2.4 & 2.0 & 3.0 & 0.0 \\
2 & 2.1 & 2.4 & 3.5 & 3.0 & 0.0 \\
3 & 2.4 & 2.4 & 3.1 & 3.0 & 0.0 \\
4 & 2.1 & 2.4 & 3.1 & 3.0 & 0.0 \\
5 & 2.1 & 2.4 & 2.0 & 3.0 & 0.0 \\
6 & 2.0 & 2.3 & 3.4 & 3.0 & 0.0 \\
7 & 2.4 & 2.3 & 3.4 & 3.0 & 0.0 \\
8 & 2.2 & 2.3 & 3.4 & 3.0 & 0.0 \\
9 & 2.4 & 2.4 & 3.5 & 3.0 & 0.0 \\
10 & 2.0 & 2.4 & 3.5 & 3.0 & 0.0 \\
\hline
\end{tabular}


Table S3. EnzyDock runs for (+)-bornyl diphosphate synthase. A single crystal water, diphosphate and three $\mathrm{Mg}^{2+}$ ions are included as cofactors. NOE restrains are imposed between ligand $\mathrm{C} 1 / \mathrm{C} 2$ carbon atoms and $\mathrm{O} 1$ oxygen atom of diphosphate, and between diphosphate and $\mathrm{Mg}^{2+}$ ions. The consensus RMSD ( $\AA$ ) is calculated relative to the ligand, (+)-bornyl diphosphate.

\begin{tabular}{cccccc}
\hline $\begin{array}{c}\text { EnzyDock Run } \\
\text { No. }\end{array}$ & $\begin{array}{c}\text { geranyl } \\
\text { diphosphate }\end{array}$ & $\begin{array}{c}\text { linalyl } \\
\text { cation }\end{array}$ & $\begin{array}{c}(4 \mathrm{R})-\alpha- \\
\text { terpinyl cation }\end{array}$ & $\begin{array}{c}\text { 2-bornyl } \\
\text { cation }\end{array}$ & $\begin{array}{c}(+) \text {-bornyl } \\
\text { diphosphate }\end{array}$ \\
\hline 1 & 2.1 & 2.6 & 2.0 & 1.6 & 0.0 \\
2 & 2.1 & 2.4 & 2.1 & 1.8 & 0.0 \\
3 & 2.0 & 2.3 & 2.3 & 2.2 & 0.0 \\
4 & 2.0 & 2.7 & 1.9 & 1.7 & 0.0 \\
5 & 2.0 & 2.3 & 2.3 & 2.2 & 0.0 \\
6 & 2.1 & 2.4 & 2.0 & 1.8 & 0.0 \\
7 & 2.1 & 2.4 & 2.0 & 1.8 & 0.0 \\
8 & 2.0 & 2.3 & 2.3 & 2.2 & 0.0 \\
9 & 2.0 & 2.3 & 2.3 & 2.2 & 0.0 \\
10 & 2.0 & 2.7 & 1.9 & 1.7 & 0.0 \\
\hline
\end{tabular}

Table S4. EnzyDock runs for (+)-bornyl diphosphate synthase. A single crystal water, diphosphate and three $\mathrm{Mg}^{2+}$ ions are included as cofactors. NOE restrains are imposed between ligand $\mathrm{C} 1 / \mathrm{C} 2$ carbon atoms and $\mathrm{O} 1$ oxygen atom of the diphosphate, and between diphosphate and $\mathrm{Mg}^{2+}$ ions. Harmonic BESTFIT restraints are applied to several carbon atoms of the ligands. The consensus RMSD ( $\AA)$ is calculated relative to the ligand, (+)-bornyl diphosphate.

\begin{tabular}{cccccc}
\hline $\begin{array}{c}\text { EnzyDock Run } \\
\text { No. }\end{array}$ & $\begin{array}{c}\text { geranyl } \\
\text { diphosphate }\end{array}$ & $\begin{array}{c}\text { linalyl } \\
\text { cation }\end{array}$ & $\begin{array}{c}\text { (4R)- } \alpha \text {-terpinyl } \\
\text { cation }\end{array}$ & $\begin{array}{c}\text { 2-bornyl } \\
\text { cation }\end{array}$ & $\begin{array}{c}\text { (+)-bornyl } \\
\text { diphosphate }\end{array}$ \\
\hline 1 & 2.1 & 1.7 & 2.4 & 2.2 & 0.0 \\
2 & 2.1 & 1.7 & 2.3 & 2.2 & 0.0 \\
3 & 2.0 & 2.0 & 3.2 & 2.2 & 0.0 \\
4 & 2.1 & 1.7 & 3.1 & 2.2 & 0.0 \\
5 & 1.9 & 1.7 & 2.4 & 2.2 & 0.0 \\
6 & 2.1 & 1.7 & 2.3 & 2.2 & 0.0 \\
7 & 2.1 & 1.7 & 2.3 & 2.2 & 0.0 \\
8 & 2.1 & 3.2 & 3.2 & 1.5 & 0.0 \\
9 & 2.1 & 1.7 & 2.3 & 2.2 & 0.0 \\
10 & 2.1 & 1.7 & 2.4 & 2.2 & 0.0 \\
\hline
\end{tabular}




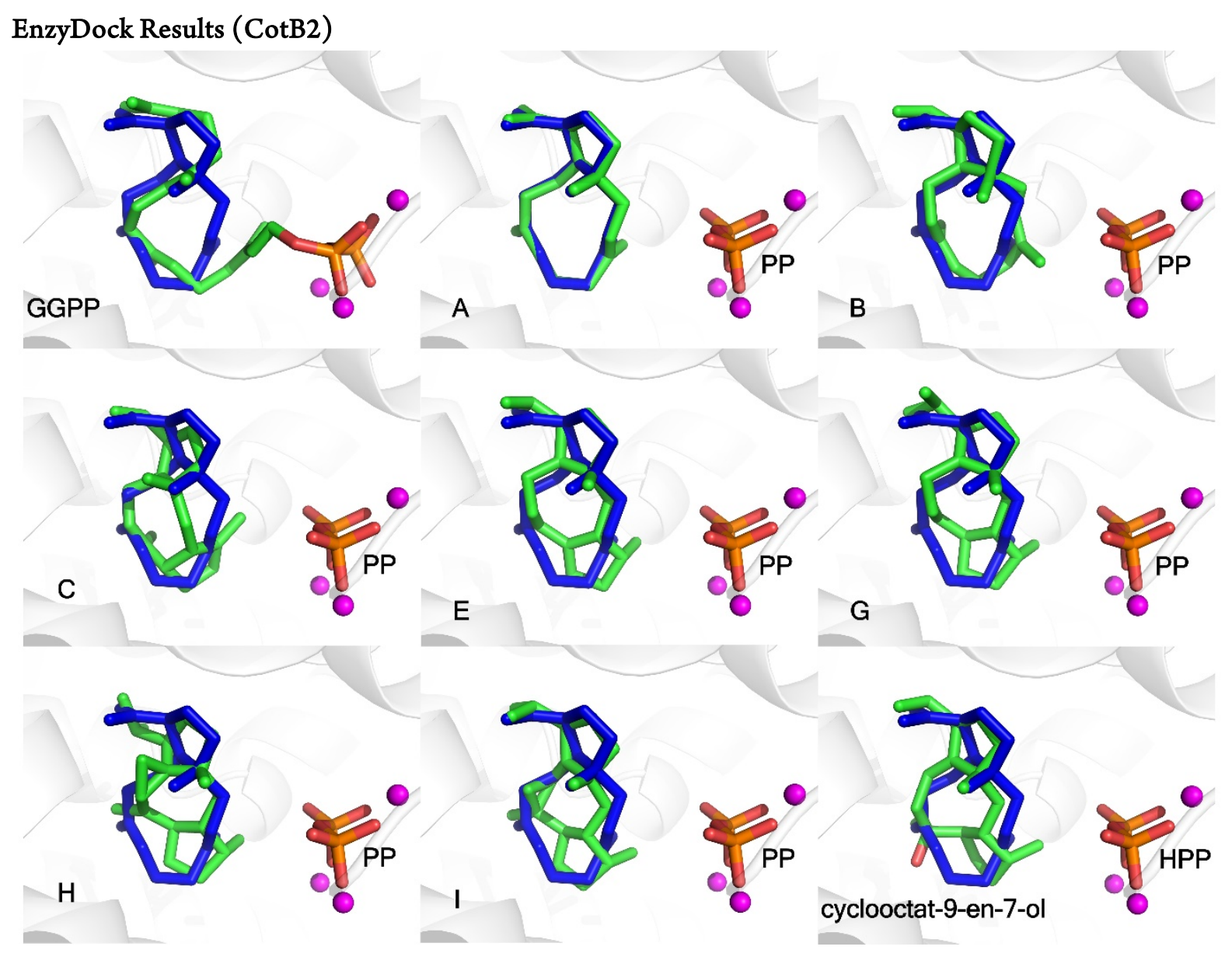

Figure S18. Overlay of poses docked with EnzyDock and the crystal structure of the diterpene cyclase CotB2. Green and blue colors represent docked poses and crystal structure, respectively. 


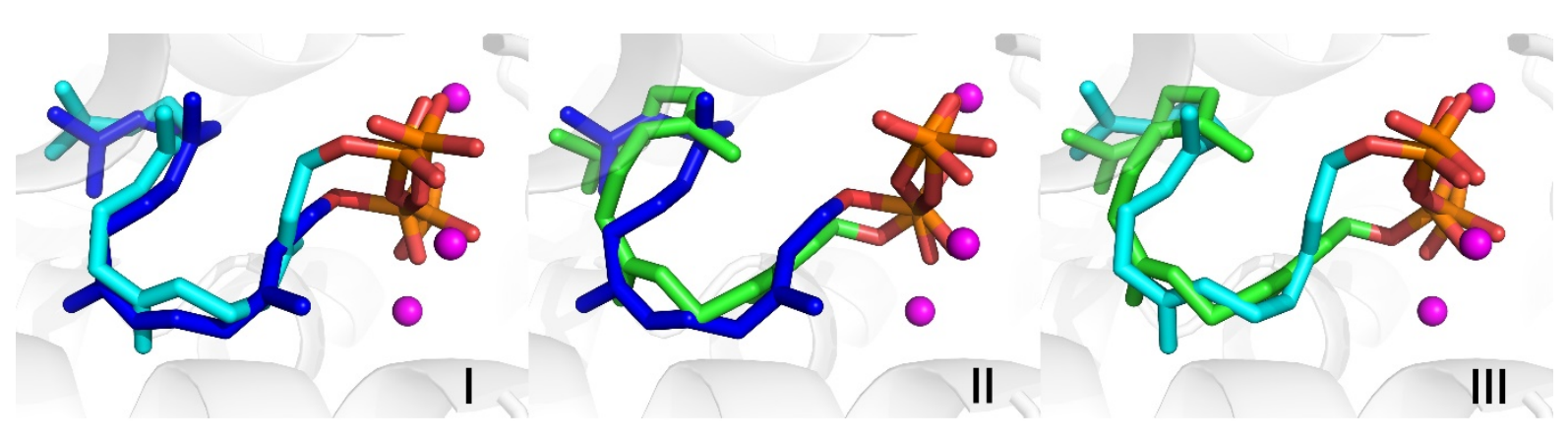

Figure S19. Overlay of the crystal structure of the diterpene cyclase, CotB2 and (I) freely docked pose (II) restrained docked pose (NOE and BESTFIT harmonic force). In (III) we show overlay of free and restrained docking poses. Cyan, green and blue colors represent freely docked pose, restrained docked pose, and crystal structure, respectively. The crystal structure (PDB code 5GUE) includes the bound substrate analog geranylgeranyl thiodiphosphate (GGSPP). ${ }^{21}$ Docking was performed with EnzyDock. 


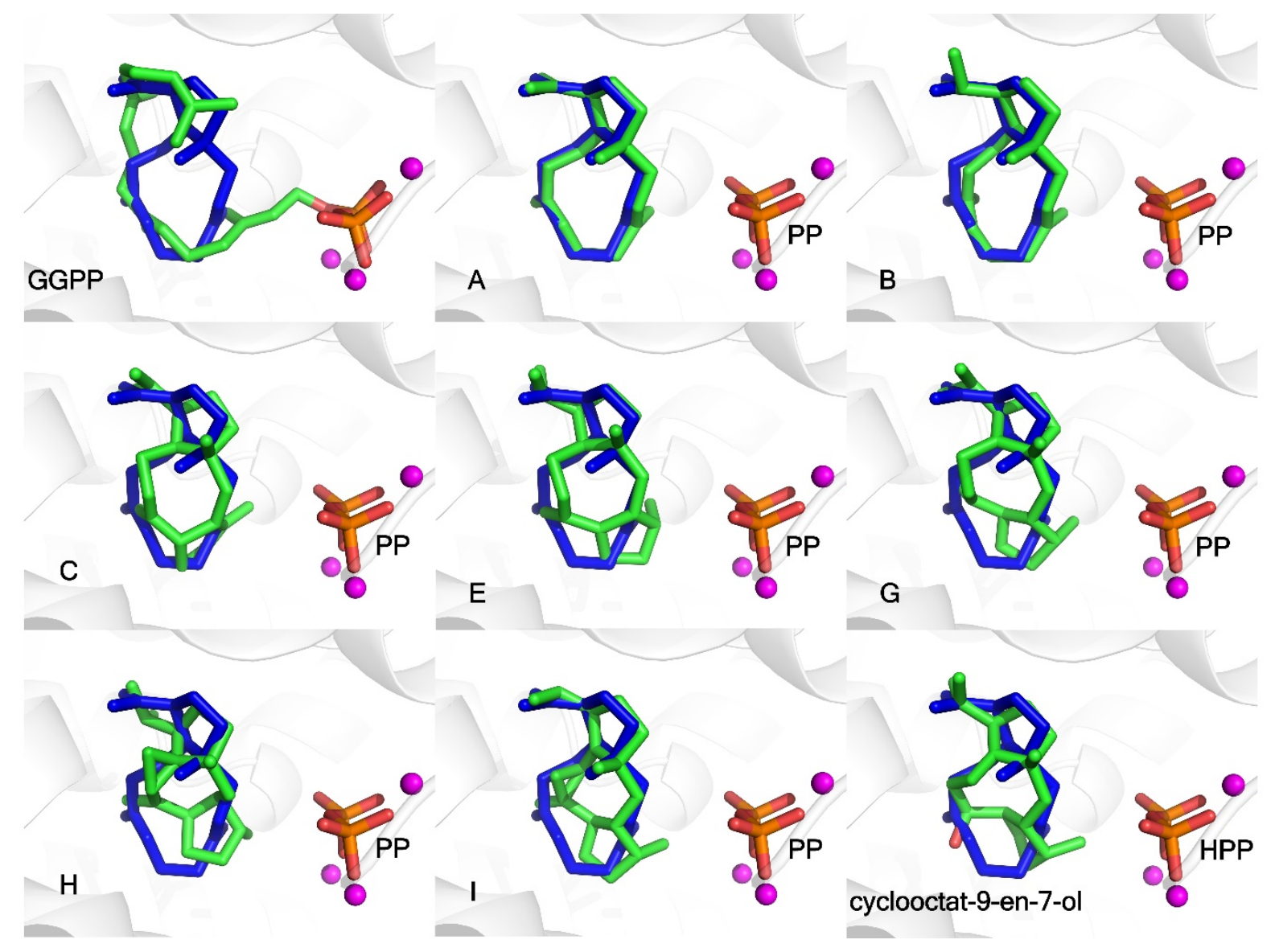

Figure S20. Overlay of poses obtained with EnzyDock and the crystal structure of the diterpene cyclase CotB2. ${ }^{5}$ NOE restrains are imposed between ligand $\mathrm{C} 1$ carbon atom and $\mathrm{O} 1 / \mathrm{O} 5$ oxygen atoms of the diphosphate, and between diphosphate and $\mathrm{Mg}^{2+}$ ions. Green and blue colors represent docked poses and crystal structure, respectively. 


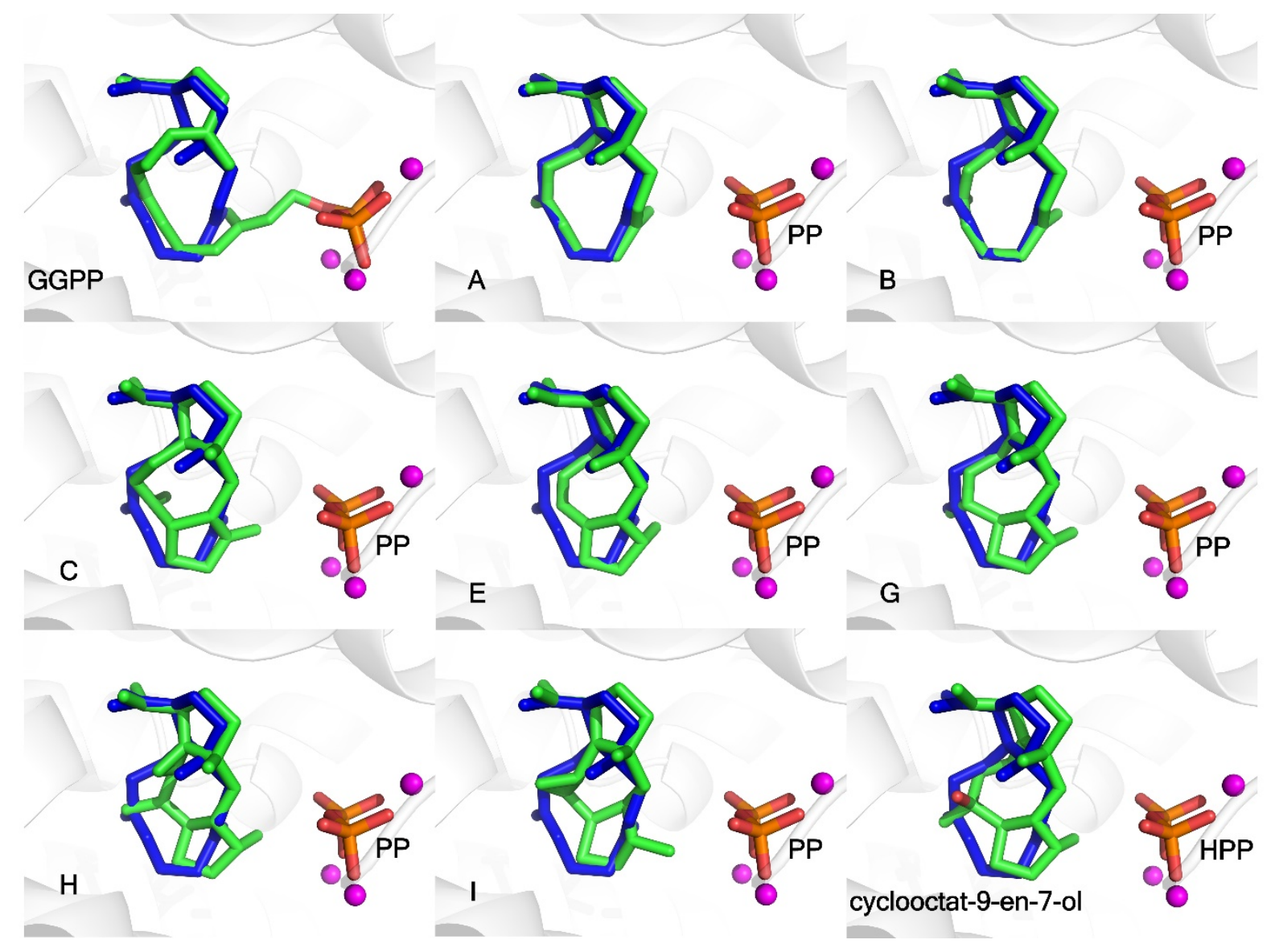

Figure S21. Overlay of poses obtained with EnzyDock and the crystal structure of the diterpene cyclase CotB2. ${ }^{5}$ NOE restrains are imposed between ligand $\mathrm{C} 1$ carbon atom and O1/O5 oxygen atoms of diphosphate, and between diphosphate and $\mathrm{Mg}^{2+}$ ions. Harmonic BESTFIT restraints are applied to several carbon atoms of the ligands. Green and blue colors represent docked pose and crystal structure, respectively. 


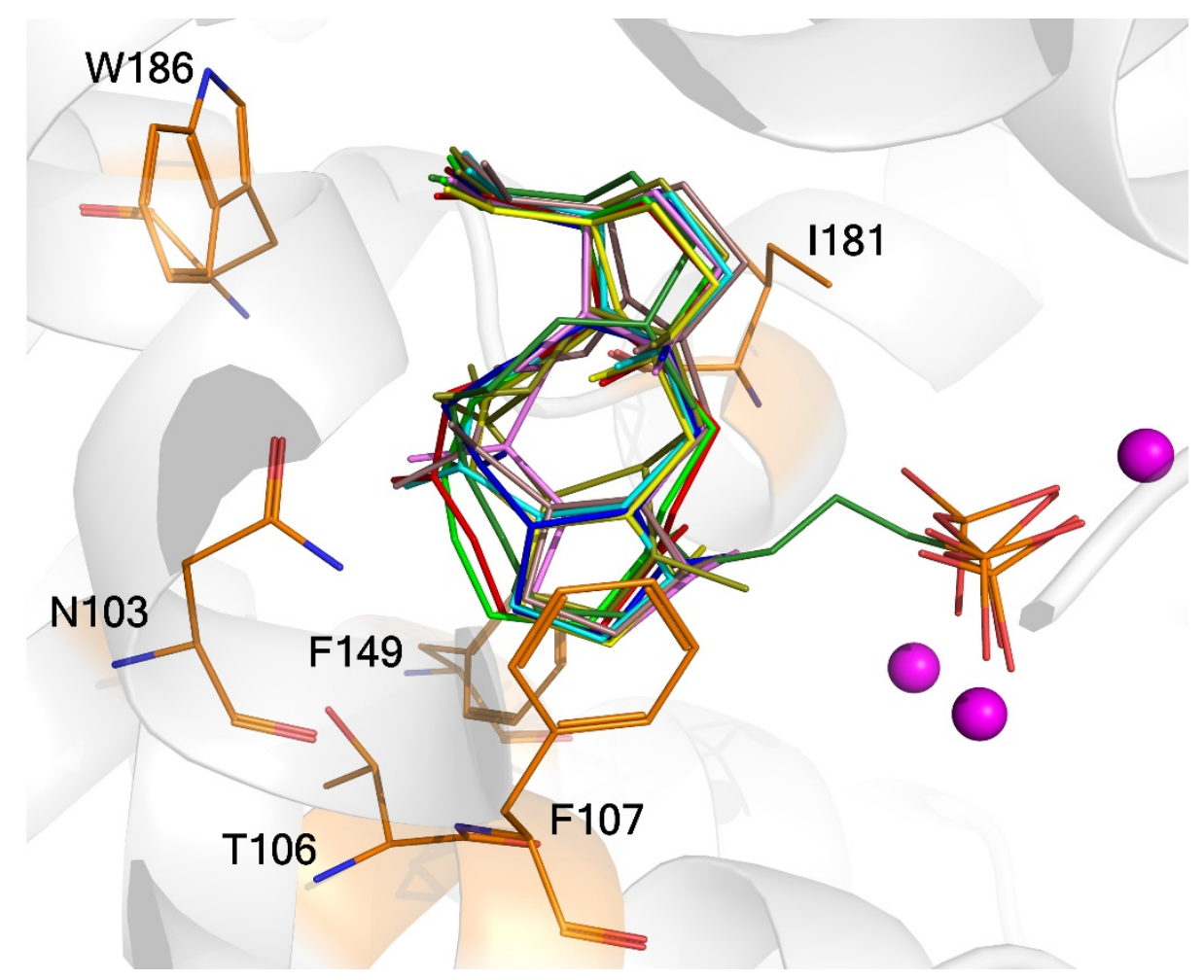

Figure S22. Overlay of all ligands (substrate, intermediates, and product) docked in the diterpene synthase, CotB2, using EnzyDock. GGPP, intermediates viz. A-I, and the product cyclooctat-9-en-7-ol, are represented by the colors forest, red, green, blue, yellow, cyan, violet, deep-olive and dirty-violet color, respectively. Magenta spheres represent $\mathrm{Mg}^{2+}$ ions. 


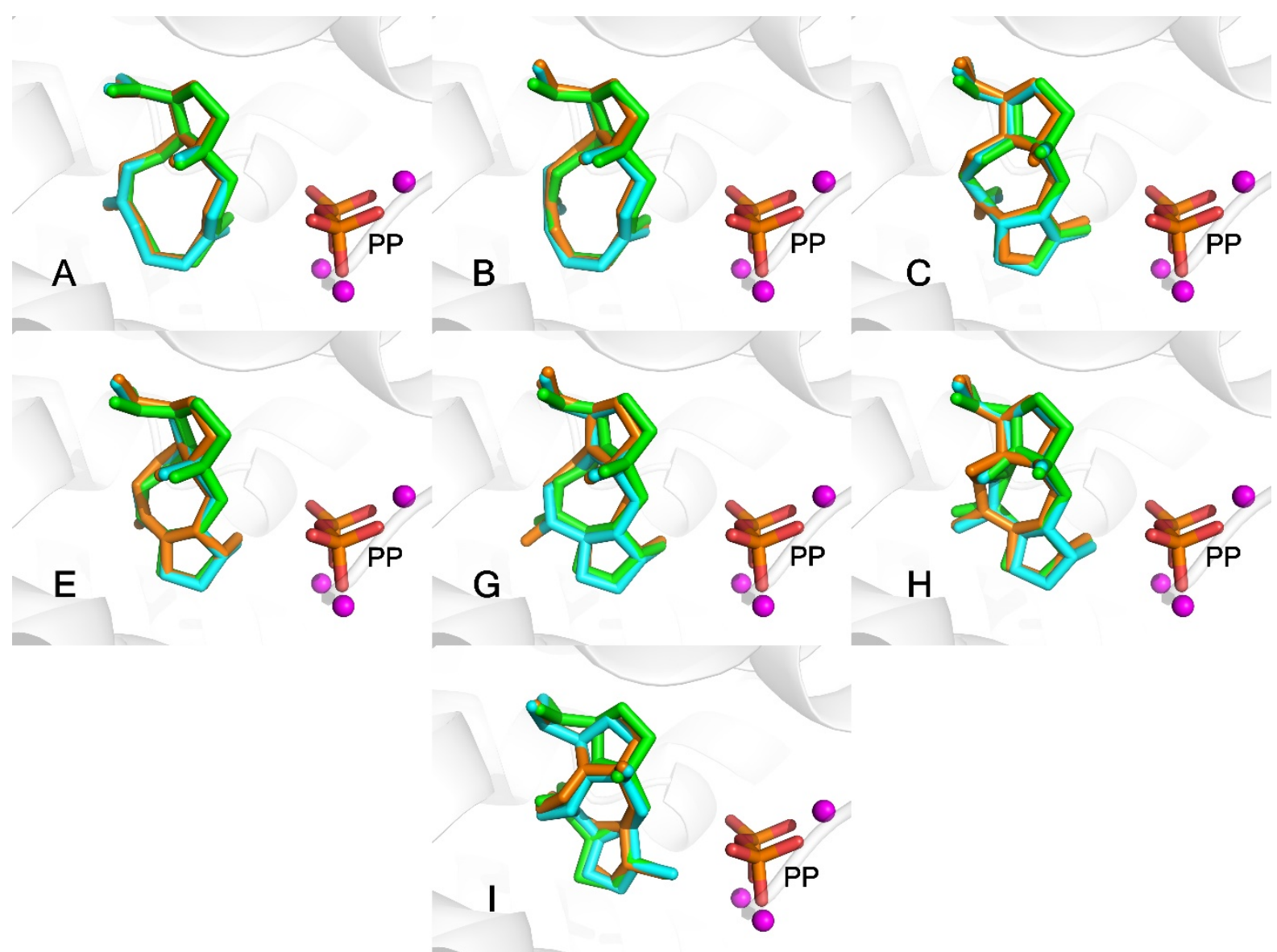

Figure S23. Overlay of docked poses obtained for in the diterpene synthase, CotB2, from EnzyDock. The docked poses were geometry optimized within grid, in full protein environment (i.e. MM without grid), and using QM/MM (no grid), and are represented by green, cyan, and orange colors, respectively. Magenta spheres represent $\mathrm{Mg}^{2+}$ ions. 


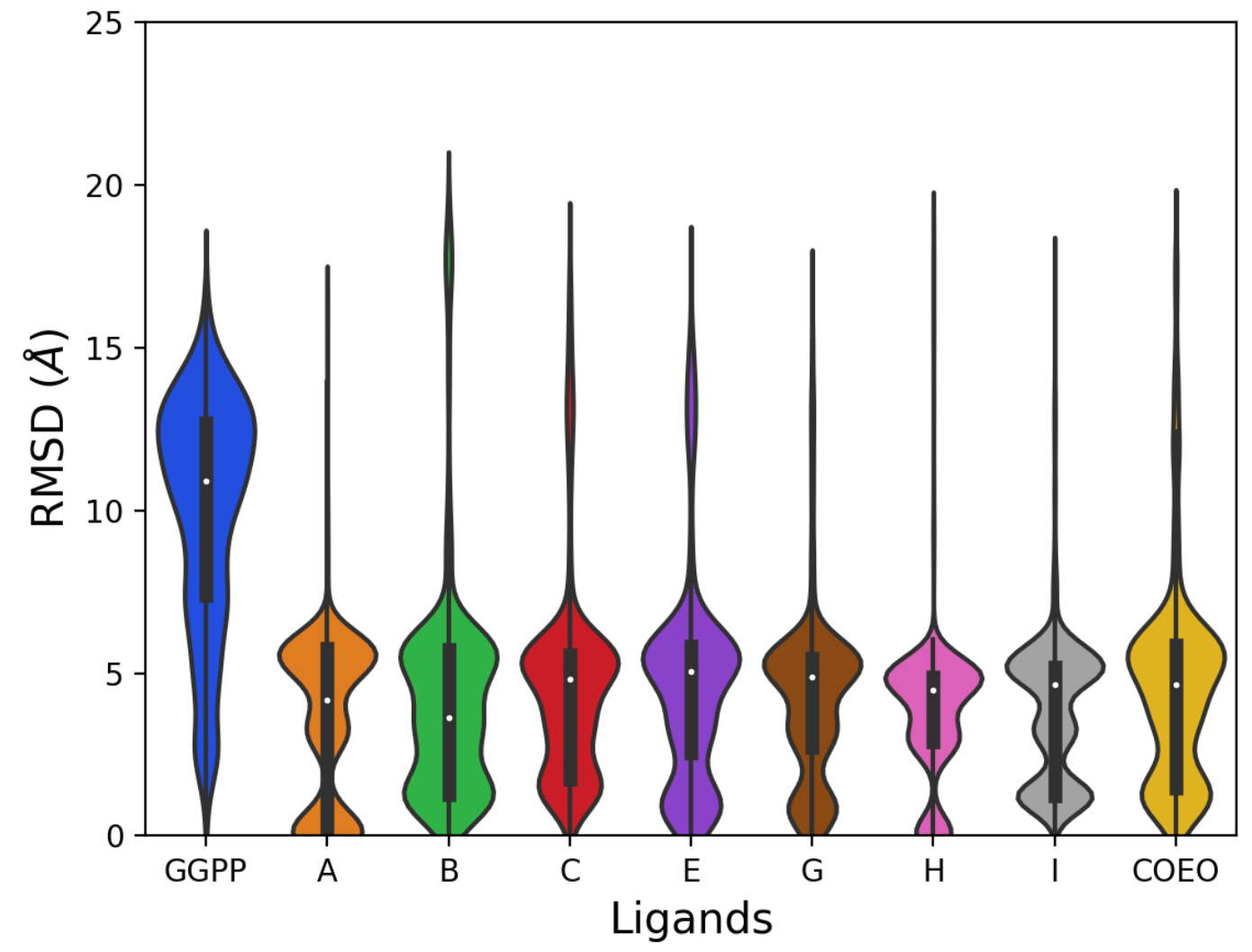

Figure S24. Violin plot of RMSD for all docked poses of the ligands in the diterpene cyclase CotB2 relative to the best docked pose (i.e. lowest EnzyDock energy). A total of 1250 docked poses for each ligand are considered. Diphosphate and three $\mathrm{Mg}^{2+}$ ions are included as cofactors. 


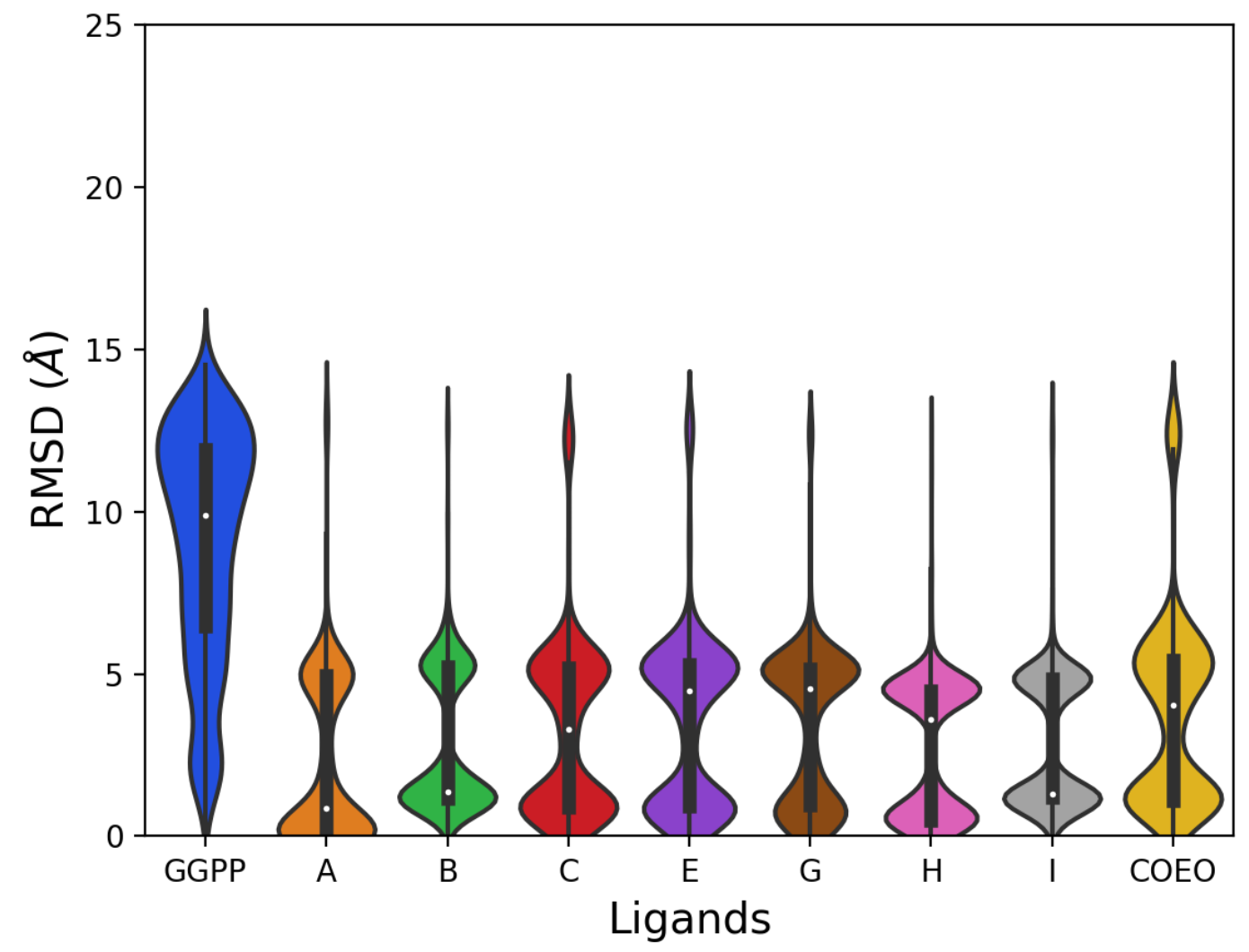

Figure S25. Violin plot of RMSD for all docked poses of the ligands in the diterpene cyclase CotB2 relative to the best docked pose (i.e. lowest EnzyDock energy). A total of 1250 docked poses for each ligand are considered. Diphosphate and three $\mathrm{Mg}^{2+}$ ions are included as cofactors. NOE restrains are imposed between ligand $\mathrm{C} 1$ carbon atom and O1/O5 oxygen atoms of the diphosphate, and between diphosphate and $\mathrm{Mg}^{2+}$ ions. 


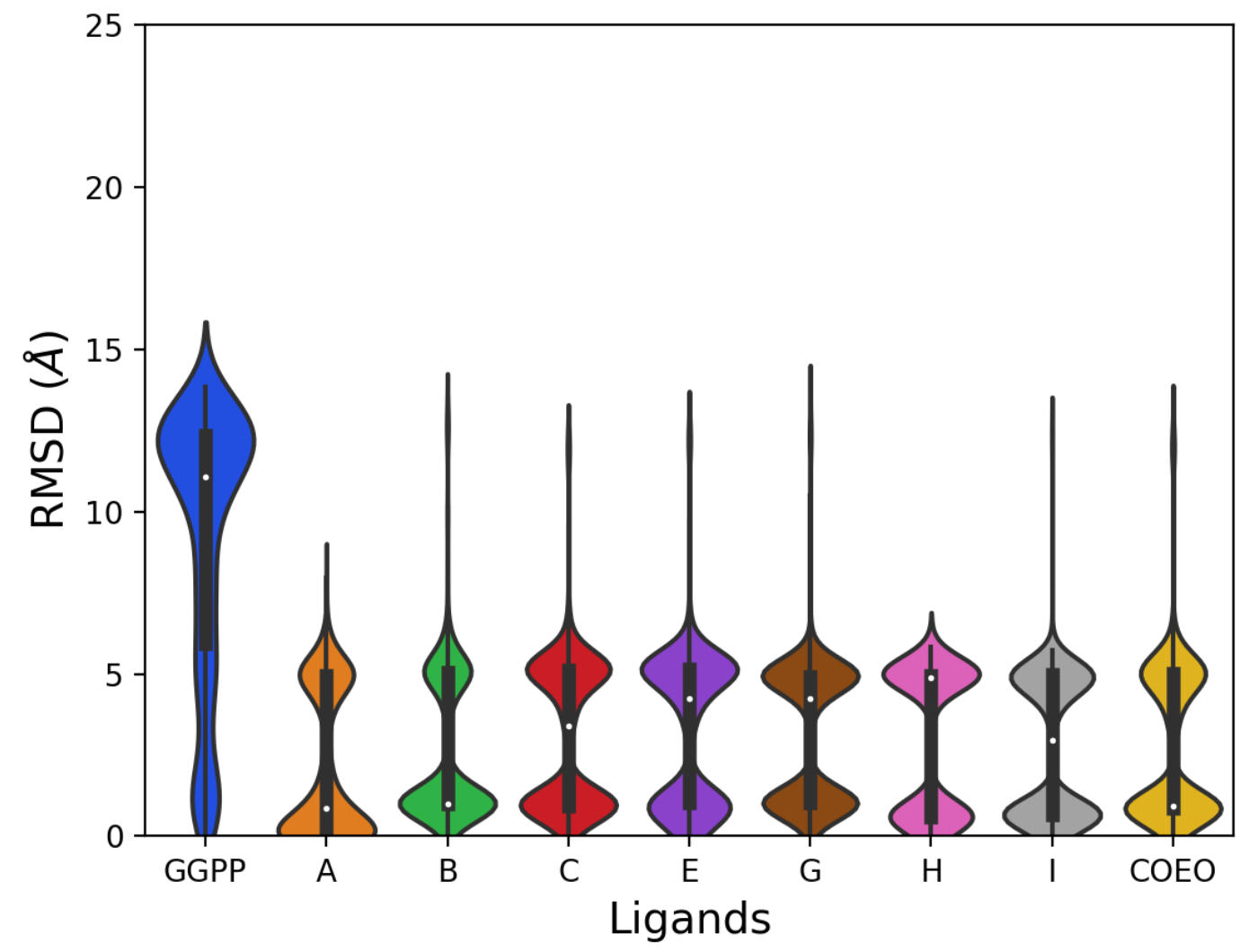

Figure S26. Violin plot of RMSD for all docked poses of the ligands in the diterpene cyclase CotB2 relative to the best docked pose (i.e. lowest EnzyDock energy). A total of 1250 docked poses for each ligand are considered. Diphosphate and three $\mathrm{Mg}^{2+}$ ions are included as cofactors. NOE restraints are imposed between ligand $\mathrm{C} 1$ carbon atom and O1/O5 oxygen atoms of the diphosphate, and between diphosphate and $\mathrm{Mg}^{2+}$ ions. Harmonic BESTFIT restraints are applied to several carbon atoms of the ligands. 
Table S5. EnzyDock runs for the diterpene cyclase CotB2. Diphosphate and three $\mathrm{Mg}^{2+}$ ions are included as cofactors.

The consensus RMSD $(\AA)$ is calculated relative to the ligand intermediate, A.

\begin{tabular}{cccccccccc}
\hline $\begin{array}{c}\text { EnzyDock } \\
\text { Run No }\end{array}$ & GGPP & A & B & C & E & G & H & I & $\begin{array}{c}\text { cyclooctat-9- } \\
\text { en-7-ol }\end{array}$ \\
\hline 1 & 1.2 & 0.0 & 1.4 & 1.5 & 1.2 & 1.3 & 1.3 & 1.2 & 1.3 \\
2 & 2.5 & 0.0 & 0.8 & 1.1 & 0.9 & 1.5 & 1.5 & 1.3 & 1.0 \\
3 & 1.3 & 0.0 & 0.5 & 1.3 & 1.2 & 1.3 & 1.3 & 1.2 & 1.3 \\
4 & 2.4 & 0.0 & 0.5 & 1.4 & 1.2 & 1.4 & 1.3 & 1.0 & 1.3 \\
5 & 1.2 & 0.0 & 0.5 & 1.3 & 1.2 & 1.3 & 1.2 & 1.2 & 1.3 \\
6 & 1.3 & 0.0 & 0.5 & 1.5 & 1.2 & 1.3 & 1.3 & 1.0 & 1.0 \\
7 & 1.2 & 0.0 & 1.2 & 1.3 & 1.2 & 1.3 & 1.3 & 1.0 & 1.0 \\
8 & 2.4 & 0.0 & 0.5 & 1.4 & 1.2 & 1.3 & 1.3 & 1.0 & 1.3 \\
9 & 2.4 & 0.0 & 0.6 & 1.3 & 1.2 & 1.3 & 1.2 & 1.0 & 1.3 \\
10 & 1.2 & 0.0 & 1.2 & 1.5 & 1.2 & 1.3 & 1.2 & 1.0 & 1.3 \\
\hline
\end{tabular}

Table S6. EnzyDock runs for the diterpene cyclase CotB2. Diphosphate and three $\mathrm{Mg}^{2+}$ ions are included as cofactors. NOE restraints are imposed between ligand $\mathrm{C} 1$ carbon atoms and O1/O5 oxygen atoms of the diphosphate, and between diphosphate and $\mathrm{Mg}^{2+}$ ions. The consensus RMSD $(\AA)$ is calculated relative to the ligand intermediate, $\mathrm{A}$.

\begin{tabular}{cccccccccc}
\hline $\begin{array}{c}\text { EnzyDock } \\
\text { Run No }\end{array}$ & GGPP & A & B & C & E & G & H & I & $\begin{array}{c}\text { cyclooctat-9- } \\
\text { en-7-ol }\end{array}$ \\
\hline 1 & 2.4 & 0.0 & 0.5 & 1.4 & 1.3 & 1.4 & 1.3 & 1.2 & 1.2 \\
2 & 2.4 & 0.0 & 1.4 & 1.4 & 1.3 & 1.4 & 1.4 & 1.2 & 1.3 \\
3 & 2.3 & 0.0 & 1.4 & 1.4 & 1.3 & 1.4 & 1.5 & 1.2 & 1.2 \\
4 & 2.3 & 0.0 & 1.4 & 1.4 & 1.3 & 1.4 & 1.5 & 1.2 & 1.4 \\
5 & 2.4 & 0.0 & 0.5 & 1.4 & 1.3 & 1.4 & 1.4 & 1.2 & 1.3 \\
6 & 2.4 & 0.0 & 0.5 & 1.4 & 1.3 & 1.4 & 1.4 & 1.2 & 1.2 \\
7 & 1.5 & 0.0 & 0.6 & 1.4 & 1.3 & 1.4 & 1.4 & 1.2 & 1.1 \\
8 & 2.4 & 0.0 & 1.4 & 1.4 & 1.3 & 1.4 & 1.4 & 1.2 & 1.3 \\
9 & 2.4 & 0.0 & 0.5 & 1.4 & 1.3 & 1.4 & 1.4 & 1.2 & 1.4 \\
10 & 3.4 & 0.0 & 0.5 & 1.4 & 1.4 & 1.4 & 1.3 & 1.3 & 1.2 \\
\hline
\end{tabular}


Table S7. EnzyDock runs for the diterpene cyclase, CotB2. Diphosphate and three $\mathrm{Mg}^{2+}$ ions are included as cofactors. NOE restrains are imposed between ligand $\mathrm{C} 1$ carbon atom and $\mathrm{O} 1 / \mathrm{O} 5$ oxygen atoms of the diphosphate, and between diphosphate and $\mathrm{Mg}^{2+}$ ions. Harmonic BESTFIT restraints are applied to several carbon atoms of the ligands. The consensus RMSD $(\AA)$ is calculated relative to the ligand, A.

\begin{tabular}{cccccccccc}
\hline $\begin{array}{c}\text { EnzyDock } \\
\text { Run No }\end{array}$ & GGPP & A & B & C & E & G & H & I & $\begin{array}{c}\text { cyclooctat-9- } \\
\text { en-7-ol }\end{array}$ \\
\hline 1 & 1.2 & 0.0 & 0.3 & 0.6 & 0.5 & 0.5 & 0.9 & 1.1 & 0.8 \\
2 & 1.2 & 0.0 & 0.3 & 0.8 & 0.5 & 0.5 & 1.0 & 1.2 & 0.8 \\
3 & 1.2 & 0.0 & 0.6 & 0.5 & 0.5 & 0.6 & 0.9 & 1.2 & 0.8 \\
4 & 1.2 & 0.0 & 0.3 & 0.5 & 0.5 & 0.6 & 0.9 & 1.2 & 0.8 \\
5 & 1.2 & 0.0 & 0.6 & 0.5 & 0.5 & 0.6 & 1.0 & 1.2 & 0.8 \\
6 & 1.2 & 0.0 & 0.3 & 0.5 & 0.5 & 0.6 & 0.9 & 0.8 & 0.8 \\
7 & 1.2 & 0.0 & 0.6 & 0.8 & 0.5 & 0.5 & 0.9 & 1.2 & 0.8 \\
8 & 1.2 & 0.0 & 0.3 & 0.8 & 0.6 & 1.1 & 0.9 & 1.2 & 0.8 \\
9 & 1.2 & 0.0 & 0.6 & 0.8 & 0.5 & 1.1 & 1.0 & 1.2 & 0.8 \\
10 & 1.2 & 0.0 & 0.3 & 0.5 & 0.5 & 0.6 & 0.9 & 1.2 & 0.8 \\
\hline
\end{tabular}




\section{EnzyDock Results (LepI)}

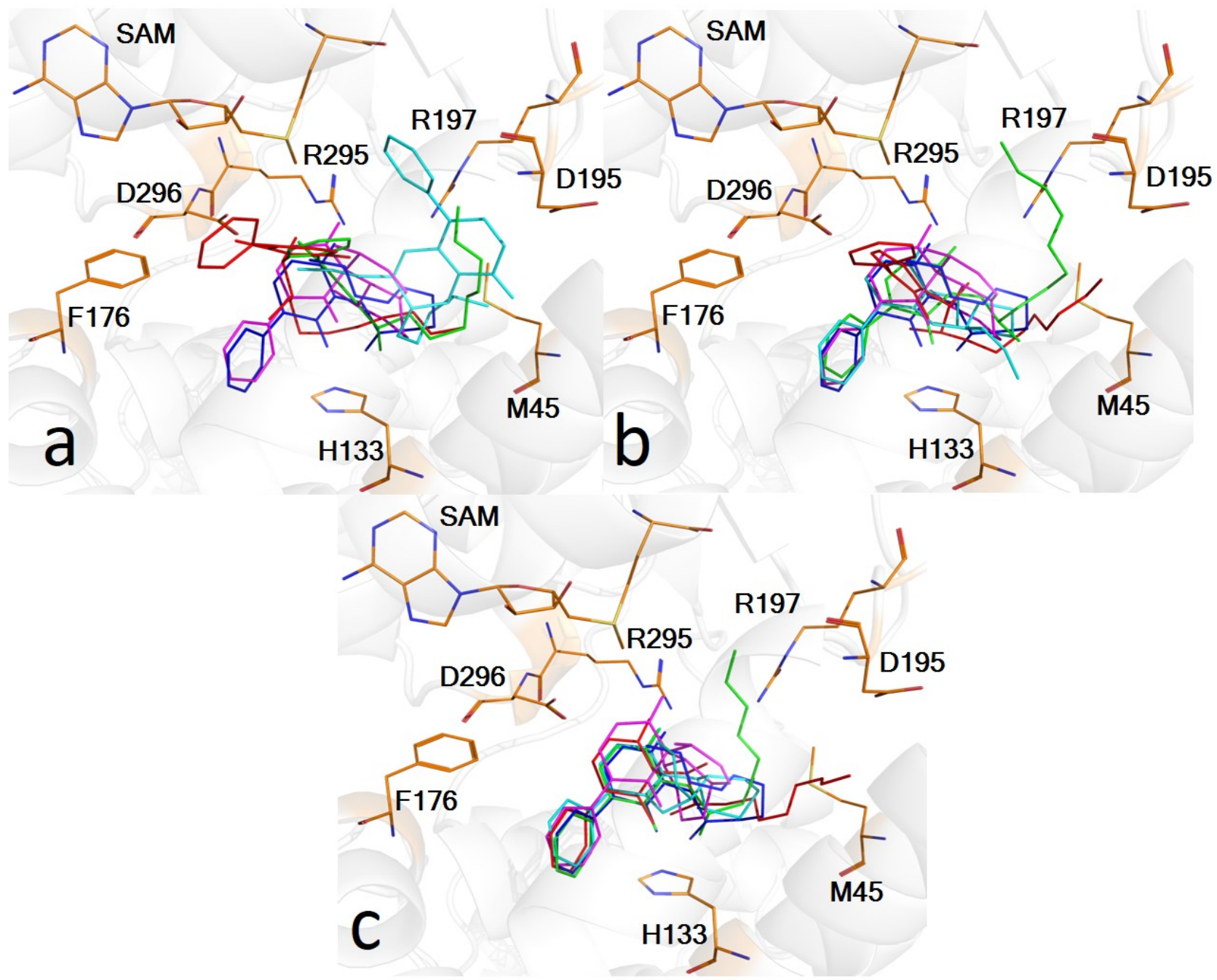

Figure S27. Overlay of all ligands (substrate, intermediate, transitions state and products) docked in LepI using EnzyDock. (a) Free docking, (b) NOE restraints are imposed between ligand O4 oxygen atom and NE2 nitrogen atom of His133, (c) NOE restraints are imposed between ligand O4 oxygen atom and NE2 nitrogen atom of His133, and harmonic BESTFIT restraints are applied to several carbon atoms and ring nitrogen atom of the ligands. Substrate (2pyridone alcohol), intermediate (E quinone methide), transition state, and products (leporin $\mathrm{C}$, pyridine dione) are represented by red, green, blue, cyan and magenta colors, respectively. 


\section{EnzyDock Results (ProR)}

Table S8. Hydrogen bond distances ( $\AA$ ) between ProR and ligand (L-Pro, D-Pro, and transition state) using flexible and fixed enzyme docking (fixed in parenthesis).

\begin{tabular}{ll|lll}
\hline Donor & Acceptor & L-Pro & Transition state & D-Pro \\
\hline N, Gly131 & OXT, Pro & $2.91(2.93)$ & $2.78(2.74)$ & $2.83(2.80)$ \\
\hline N, His132 & O, Pro & $3.03(2.90)$ & $2.79(2.86)$ & $2.82(2.76)$ \\
\hline N, Gly301 & O, Pro & $2.87(2.79)$ & $2.77(2.73)$ & $2.89(2.99)$ \\
\hline N, Thr302 & OXT, Pro & $2.77(2.80)$ & $2.82(2.92)$ & $3.11(2.98)$ \\
\hline OG1, Thr302 & OXT, Pro & $2.63(2.93)$ & $2.63(2.81)$ & $2.73(2.84)$ \\
\hline N, Pro & ND1, His132 & $3.08(2.94)$ & $2.97(2.94)$ & $2.84(2.90)$ \\
\hline N, Pro & OD2, Asp296 & $2.95(2.79)$ & $2.82(2.82)$ & $2.79(2.78)$ \\
\hline
\end{tabular}




\section{EnzyDock Results (Cruzain)}

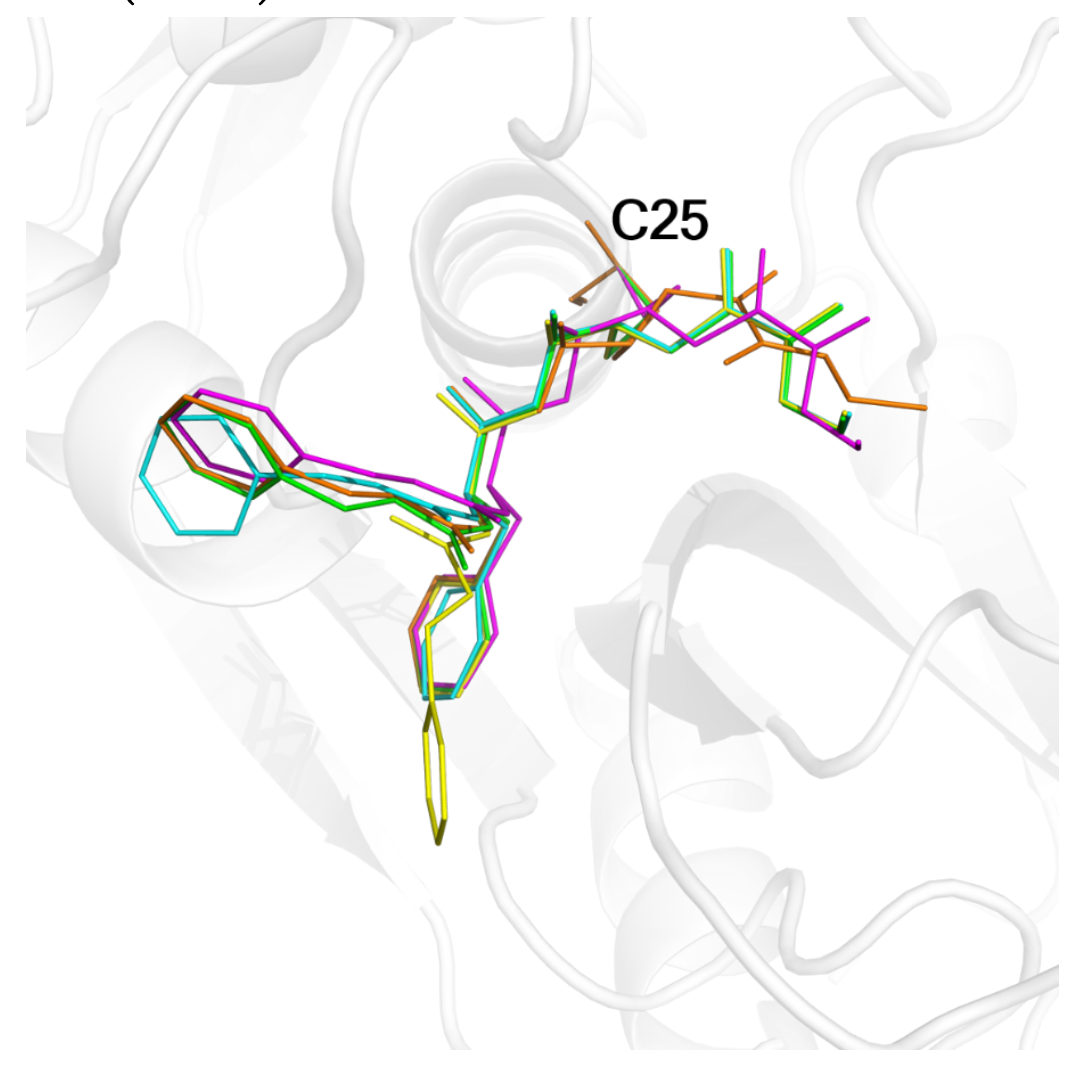

Figure S28. Overlay of the best and other four highest ranking poses of the covalently bonded ligand docked in the cysteine protease cruzain, using EnzyDock. Green color represents the best pose and the other four docked poses are represented by cyan, magenta, yellow, and orange color, respectively.

Table S9. RMSD $(\AA)$ values for the five best EnzyDock docked poses relative to the crystal structure in cruzain. ${ }^{9}$

\begin{tabular}{llllll}
\hline $\begin{array}{l}\text { EnzyDock } \\
\text { pose No. }\end{array}$ & pose 1 & pose 2 & pose 3 & pose 4 & pose 5 \\
\hline RMSD & 1.5 & 1.3 & 1.7 & 4.2 & 1.7 \\
\hline
\end{tabular}




\section{References:}

1. $\quad$ Friesner, R. A.; Banks, J. L.; Murphy, R. B.; Halgren, T. A.; Klicic, J. J.; Mainz, D. T.; Repasky, M. P.; Knoll, E. H.; Shelley, M.; Perry, J. K.; et al., Glide: a new approach for rapid, accurate docking and scoring. 1. Method and assessment of docking accuracy.J. Med. Chem. 2004, 47, 1739-1749.

2. $\quad$ Friesner, R. A.; Murphy, R. B.; Repasky, M. P.; Frye, L. L.; Greenwood, J. R.; Halgren, T. A.; Sanschagrin, P. C.; Mainz, D. T., Extra precision glide: docking and scoring incorporating a model of hydrophobic enclosure for protein-ligand complexes. J. Med. Chem. 2006, 49, 6177-6196.

3. Halgren, T. A.; Murphy, R. B.; Friesner, R. A.; Beard, H. S.; Frye, L. L.; Pollard, W. T.; Banks, J. L., Glide: a new approach for rapid, accurate docking and scoring. 2. Enrichment factors in database screening. J. Med. Chem. 2004, 47, 1750-1759.

4. Weitman, M.; Major, D. T., Challenges posed to bornyl diphosphate synthase: diverging reaction mechanisms in monoterpenes. J. Am. Chem. Soc. 2010, 132, 6349-6360.

5. $\quad$ Driller, R.; Janke, S.; Fuchs, M.; Warner, E.; Mhashal, A. R.; Major, D. T.; Christmann, M.; Bruck, T.; Loll, B., Towards a comprehensive understanding of the structural dynamics of a bacterial diterpene synthase during catalysis. Nat. Commun. 2018, 9, 3971.

6. $\quad$ Byrne, M. J.; Lees, N. R.; Han, L. C.; van der Kamp, M. W.; Mulholland, A. J.; Stach, J. E.; Willis, C. L.; Race, P. R., The Catalytic Mechanism of a Natural Diels-Alderase Revealed in Molecular Detail. J. Am. Chem. Soc. 2016, 138, 6095-6098.

7. $\quad$ Chang, Z.; Ansbacher, T.; Zhang, L.; Yang, Y.; Ko, T.-P.; Zhang, G.; Liu, W.; Huang, J.-W.; Dai, L.; Guo, R.-T.; et al., Crystal structure of LepI, a multifunctional SAM-dependent enzyme which catalyzes pericyclic reactions in leporin biosynthesis. Org. Biomol. Chem. 2019, 17, 2070-2076.

8. Zhu, K.; Borrelli, K. W.; Greenwood, J. R.; Day, T.; Abel, R.; Farid, R. S.; Harder, E., Docking covalent inhibitors: a parameter free approach to pose prediction and scoring. J. Chem. Inf. Model. 2014, 54, 1932-1940.

9. $\quad$ Choe, Y.; Brinen, L. S.; Price, M. S.; Engel, J. C.; Lange, M.; Grisostomi, C.; Weston, S. G.; Pallai, P. V.; Cheng, H.; Hardy, L. W.; et al., Development of $\alpha$-keto-based inhibitors of cruzain, a cysteine protease implicated in Chagas disease. Bioorg. Med. Chem. 2005, 13, 2141-2156.

10. Sastry, G. M.; Adzhigirey, M.; Day, T.; Annabhimoju, R.; Sherman, W., Protein and ligand preparation: parameters, protocols, and influence on virtual screening enrichments. J. Comput. Aided Mol. Des. 2013, 27, 221-234.

11. Schrödinger Release 2019-1: LigPrep, S., LLC, New York, NY, 2019.

12. Repasky, M. P.; Murphy, R. B.; Banks, J. L.; Greenwood, J. R.; Tubert-Brohman, I.; Bhat, S.; Friesner, R. A., Docking performance of the glide program as evaluated on the Astex and DUD datasets: a complete set of glide SP results and selected results for a new scoring function integrating WaterMap and glide. J. Comput. Aid. Mol. Des. 2012, 26, 787-799.

13. Frisch, M. J.; Trucks, G. W.; Schlegel, H. B.; Scuseria, G. E.; Robb, M. A.; Cheeseman, J. R.; Scalmani, G.; Barone, V.; Petersson, G. A.; Nakatsuji, H.; et al. Gaussian 16, revision A. 03, 2016.

14. Becke, A. D., Density-functional thermochemistry. V. Systematic optimization of exchangecorrelation functionals. J. Chem. Phys. 1997, 107, 8554-8560.

15. Grimme, S., Semiempirical GGA-type density functional constructed with a long-range dispersion correction. J. Comput. Chem. 2006, 27, 1787-1799.

16. Hariharan, P. C.; Pople, J. A., Influence of polarization functions on molecular-orbital hydrogenation energies. Theor. Chem. Acc. 1973, 28, 213-222.

17. Clark, T.; Chandrasekhar, J.; Spitznagel, G. W.; Schleyer, P. v. R., Efficient diffuse functionaugmented basis-sets for anion calculations. 3. The 3-21+G basis set for 1st-row elements, Li-F. J. Comput. Chem. 1983, 4, 294-301. 
18. Harder, E.; Damm, W.; Maple, J.; Wu, C.; Reboul, M.; Xiang, J. Y.; Wang, L.; Lupyan, D.; Dahlgren, M. K.; Knight, J. L.; et al., OPLS3: A Force Field Providing Broad Coverage of Drug-like Small Molecules and Proteins. J. Chem. Theory Comput. 2016, 12, 281-296.

19. Pagadala, N. S.; Syed, K.; Tuszynski, J., Software for molecular docking: a review. Biophys. Rev. 2017, 9, 91-102.

20. Whittington, D. A.; Wise, M. L.; Urbansky, M.; Coates, R. M.; Croteau, R. B.; Christianson, D. W., Bornyl diphosphate synthase: structure and strategy for carbocation manipulation by a terpenoid cyclase. Proc. Natl. Acad. Sci. U. S. A. 2002, 99, 15375-15380.

21. Tomita, T.; Kim, S.-Y.; Teramoto, K.; Meguro, A.; Ozaki, T.; Yoshida, A.; Motoyoshi, Y.; Mori, N.; Ishigami, K.; Watanabe, H.; et al., Structural Insights into the CotB2-catalyzed cyclization of geranylgeranyl diphosphate to the diterpene cyclooctat-9-en-7-ol. ACS Chem. Biol.2017, 12, 1621-1628. 